nephron

Experimental

Nephrology

and Genetics
Nephron 2018;139:83-111 DOI: $10.1159 / 000488293$

Not available in print, free online access only:

www.karger.com/Article/Abstract/488293

\title{
Abstracts
}

\section{0th Meeting of the European Renal Cell Study Group (ERCSG)}

April 19-22, 2018, Florence, Italy

\author{
Editors \\ Paola Romagnani, Florence \\ Elena Lazzeri, Florence \\ Laura Lasagni, Florence \\ Marco Allinovi, Florence \\ Benedetta Mazzinghi, Florence \\ Francesca Becherucci, Florence \\ Letizia De Chiara, Florence
}




\section{Organizing Secretariat}

MCR Conference Srl

Via Finlandia, 26

IT-50126 Florence (Italy) +390554364475

info@mcrconference.it www.mcrconference.it
S. Karger

Medical and Scientific Publishers Basel · Freiburg · Paris · London New York · Chennai · New Delhi Bangkok · Beijing · Shanghai · Tokyo . Kuala Lumpur · Singapore · Sydney
Disclaimer

The statements, opinions and data contained in this publication are solely those of the individual authors and contributors and not of the publisher and the editor(s). The appearance of advertisements in the journal is not a warranty, endorsement, or approval of the products or services advertised or of their effectiveness, quality or safety. The publisher and the editor(s) disclaim responsibility for any injury to persons or property resulting from any ideas, methods, instructions or products referred to in the content or advertisements.

Drug Dosage

The authors and the publisher have exerted every effort to ensure that drug selection and dosage set forth in this text are in accord with current recommendations and practice at the time of publication. However, in view of ongoing research, changes in government regulations, and the constant flow of information relating to drug therapy and drug reactions, the reader is urged to check the package insert for each drug for any change in indications and dosage and for added warnings and precautions. This is particularly important when the recommended agent is a new and/or infrequently employed drug.
All rights reserved

No part of this publication may be translated into other languages, reproduced or utilized in any form or by any means, electronic or mechanical, including photocopying, recording microcopying, or by any information storage and retrieval system, without permission in writing from the publisher or in the case of photocopying, direct payment of a specified fee to the Copyright Clearance Center (see 'General Information')

(C) Copyright 2018 by S. Karger AG

P.O. Box, CH-4009 Basel (Switzerland)

\section{KARGER}




\section{Preface}

\section{nephron \\ Experimental \\ Nephrology \\ and Genetics}

The first European Renal Cell Study Group (ERCSG) meeting was held in Gregynog Hall (Wales) in 1989. The conference was dedicated to bringing together junior and senior researchers from allover Europe to discuss molecular aspects of renal physiology and pathology. Thanks to the possibility to share research work, engage in stimulating discussions and exchange ideas, everything in an informal way, the ERCSG meeting became since then a successful annual appointment.

This year, the organizers of the 30th ERCSG have tried their best to put together an attractive program that highlights the frontiers of new knowledge, much of it yet unpublished, and combine this with clinical relevance and guidance for future research directions. With so many upcoming technical innovations and strategies for modelling of kidney disease moving from $2 \mathrm{D}$ to $3 \mathrm{D}$, such as single cell sequencing, advanced microscopy, omics, iPS-derived renal cells and organoids, we have tried to build a meeting that could face the challenge of the new times remaining in the pathway of tradition. Accordingly, the program encompasses a large spectrum of topics relevant to glomerular and tubular functional and structural integrity in health and disease, as well as to the latest strategies for building organoids, for stem cell treatments. We also look forward to lively discussions that will attempt to delineate research challenges, formulate testable hypotheses, and suggest models that can guide future research and therapeutic discovery. With the accelerated, web-centered science we face today it is needed to find sufficent time to sit together and open discussion across educational levels. Therefore, we are especially grateful to all of the participants from numerous European countries, that will ensure a stimulating scientific exchange. We are also grateful for the continued alliance between different generations of kidney researchers, as well as for the pleasure of a seamless interaction in order to start from the past to build the future of nephrology.

With many thanks,
Paola Romagnani, Excellence Centre for Research, Transfer and High Education for the development of DE NOVO Therapies (DENOTHE), University of Florence, Department of Biomedical, Experimental and Clinical Sciences "Mario Serio", University of Florence, Nephrology Unit, Meyer Children’s University Hospital, Florence

Elena Lazzeri, Excellence Centre for Research, Transfer and High Education for the development of DE NOVO Therapies (DENOTHE), University of Florence, Department of Biomedical, Experimental and Clinical Sciences "Mario Serio", University of Florence

Laura Lasagni, Excellence Centre for Research, Transfer and High Education for the development of DE NOVO Therapies (DENOTHE), University of Florence, Department of Biomedical, Experimental and Clinical Sciences "Mario Serio", University of Florence

Marco Allinovi, Excellence Centre for Research, Transfer and High Education for the development of DE NOVO Therapies (DENOTHE), University of Florence, Department of Biomedical, Experimental and Clinical Sciences "Mario Serio", University of Florence

Benedetta Mazzinghi, Nephrology Unit, Meyer Children's University Hospital, Florence

Francesca Becherucci, Nephrology Unit, Meyer Children's University Hospital, Florence

Letizia De Chiara, Excellence Centre for Research, Transfer and High Education for the development of DE NOVO Therapies (DENOTHE), University of Florence, Department of Biomedical, Experimental and Clinical Sciences "Mario Serio", University of Florence 


\section{nephron

\section{1 \\ Activation of Periostin Promotes Renal Repair in Ischemia/Reperfusion-Induced Acute Kidney Injury}

Niki Prakoura ${ }^{1}$, Raphaël Kormann 1,2, Panagiotis Kavvadas' ${ }^{1}$, Sandrine Placier ${ }^{1}$, Jean-Claude Dussaule ${ }^{1,2}$, Christos Chadjichristos ${ }^{1,2}$, Christos Chatziantoniou ${ }^{1,2}$

${ }^{1}$ INSERM UMRS 1155, Tenon Hospital, Paris, France;

${ }^{2}$ Sorbonne Université, Paris, France

Periostin, an extracellular matrix protein described as a major trigger of phenotype changes of myofibroblasts and cardiac remodelling, was found to be highly induced in the diseased kidney promoting the development of inflammation and fibrosis in different models of chronic kidney disease; however, the role of periostin in acute kidney injury (AKI) has not been studied yet. Interestingly, periostin was described to play a protective role in the heart after acute myocardial infarction. The aim of the current study was to investigate the potentially differential role of periostin during acute injury and repair of the kidney.

To examine the role of periostin in AKI we applied the model of unilateral renal ischemia/reperfusion (I/R) with contralateral nephrectomy in mice with deletion or conditional overexpression of periostin in the kidney. We examined two time points of reperfusion following ischemia: $24 \mathrm{~h}$ which corresponds to the peak of injury, and $72 \mathrm{~h}$ which marks the repair phase. To compare the disease phenotype between the groups, measurements of creatininemia and uremia, analysis of renal structure, macrophage infiltration, tubular proliferation and apoptosis and examination of the levels of periostin, kidney injury molecules and inflammatory mediators were performed.

Periostin was found to be de novo expressed by tubular epithelial cells in the $\mathrm{I} / \mathrm{R}$ model starting at $24 \mathrm{~h}$ with its expression further augmenting at $72 \mathrm{~h}$. Mice conditionally overexpressing periostin by tubular cells (Pax8-rtTA) at the time of injury displayed significantly lower values of plasma creatinine and urea and a better preservation of kidney structure at both time points. The expression of NGAL and KIM-1, two well-known markers of kidney injury, as well as this of several pro-inflammatory mediators such as IL-6, MCP-1, and VCAM-1 was reduced in mice with conditional overexpression of periostin. PAS and TUNEL staining revealed decreased tubular necrosis/apoptosis in mice overexpressing periostin after $\mathrm{I} / \mathrm{R}$, while cell cycle arrest markers (p21Cip1, p16Ink4) were also reduced. Interestingly, macrophages were more abundant in the kidneys of periostinoverexpressing mice after injury, indicating a potential pro-reparative phenotype of macrophages induced by periostin. In accordance with these results, periostin knock-out mice showed deterioration of renal function as evidenced by increased levels of plasma creatinine and urea, enhanced expression of KIM-1 accompanied by worsened kidney structure, increased tubular apoptosis/cell-cycle arrest and reduced macrophage infiltration.
In conclusion, these findings suggest that periostin assists in renal repair after AKI, indicating the differential role of periostin in acute vs. chronic renal diseases.

\section{2 \\ Deletion of Periostin Enhances Tubular Injury and Reduces Macrophage Infiltration in Ischemia/ Reperfusion-Induced Acute Kidney Injury \\ Raphaël Kormann ${ }^{1,2}$, Niki Prakoura', Sandrine Placier', Sophie Vandermeersch ${ }^{1}$, Marie-Christine Verpont ${ }^{1}$, Jean-Claude Dussaule ${ }^{1,2}$, Christos Chadjichristos ${ }^{1,2}$, Christos Chatziantoniou ${ }^{1,2}$ \\ 'INSERM UMRS 1155, Tenon Hospital, Paris, France; \\ ${ }^{2}$ Sorbonne Université, Paris, France}

Background: Periostin has been recently identified as an important factor in the development of chronic kidney disease. We have previously demonstrated that in the hypertensive LNAMEinduced renal injury and unilateral ureteral obstruction, periostin was de novo expressed by injured tubular epithelial cells. The genetic or pharmacologic inhibition of periostin decreased tubular injury, macrophages and $\mathrm{T}$ lymphocytes infiltration, and kidney fibrosis in these models. In vitro, periostin induced collagen expression, and phosphorylation of FAK, p38 MAPK and ERK in human collecting duct cells. Therefore, we hypothesized that periostin could also be de novo induced in acute kidney injury around damaged tubular epithelial cells, and may promote tubular cell injury and local inflammation.

Methods: Unilateral renal ischemia-reperfusion (I/R) was performed in wild-type and global knock-out (KO) periostin C57/B6 mice, with four time points of reperfusion: 1, 3, 7 and 42 days. In the end of each time-point, we performed analysis of renal structure and function, measurements of macrophage infiltration and tubular apoptosis as well as examination of the expression of periostin, kidney injury and inflammatory markers.

Results: Following I/R, periostin was de novo induced especially in, but not restricted to, proximal tubular epithelial cells at the corticomedullary junction, starting at day 1 , and this overexpression persisted in all later time-points. Plasma creatinine and urea were slightly increased at day 1 in periostin KO mice, but no strain difference was observed regarding kidney structure at this time point. However, repair was delayed at day 3 in periostin KO mice, with increased plasma creatinine and urea, and persistent tubular injury. At day 7, both groups were comparable regarding kidney structure and function. At 42 days, chronic kidney failure and fibrosis (evaluated by Sirius Red coloration) were more severe in KO mice. Regarding changes in tubular epithelial cell phenotype, the expression of KIM-1 was higher at day 1 in KO mice, and 
TUNEL and cleaved caspase 3 staining revealed increased apoptosis at day 1 . In addition, the activation of cleaved caspase 3 persisted at later time points in periostin $\mathrm{KO}$ mice, in combination with increased Bax and decreased phospho-AKT, suggesting activation of pro-survival pathways in epithelial tubular cells by periostin. Electronic microscopy showed increased mitochondrial damage in proximal tubular epithelial cells of KO mice. In subsequent in vitro experiments, primary tubular cells isolated from periostin $\mathrm{KO}$ mice were more sensitive to hydrogen peroxide treatment. To note that wild-type mice displayed increased polymorphonuclear staining at day 1 , and macrophage infiltration at 3, 7 and 42 days. Most of these macrophages corresponded to M2 (alternatively activated) phenotype in wild type mice, whereas the CD206 marker expression was reduced in KO mice.

Conclusions: Periostin is induced after I/R to protect tubular epithelial cells from apoptosis, and to enhance polarization of macrophages. The final outcome is a protection against the long term consequences of developing kidney fibrosis after AKI.

\section{3}

\section{The Protective Role of Macrophage Migration Inhibitory Factor (MIF) in Acute Kidney Injury after Cardiac Surgery}

\author{
S. Djudjaj $^{1 \dagger}$, C. Stoppe ${ }^{1 \dagger}$, L. Averdunk $^{\dagger}$, A. Goetzenich ${ }^{1}$, \\ J. Soppert ${ }^{2}$, A. Marlier ${ }^{2}$, S. Kraemer ${ }^{1}$, M. Coburn ${ }^{1}$, A. Stevanovic ${ }^{1}$, \\ B.S. Kim ${ }^{1}$, G. Marx' ${ }^{1}$, S. Rex ${ }^{3}$, A. Ochi ${ }^{2}$, L. Leng ${ }^{2}$, G. Moeckel', \\ A. Linkermann ${ }^{4}$, O. El Bounkari ${ }^{5}$, A. Zarbock ${ }^{5}$, J. Bernhagen ${ }^{5}$, \\ R. Bucala ${ }^{2 \ddagger}$, P. Boor ${ }^{1 \ddagger}$ \\ ${ }^{1}$ University Hospital, RWTH Aachen, Germany; ${ }^{2}$ Yale University \\ School of Medicine, New Haven, USA; ${ }^{3}$ University Hospitals \\ Leuven, Leuven, Belgium; ${ }^{4}$ University Hospital Carl Gustav \\ Carus at the Technische Universitat Dresden, Dresden, \\ Germany; ${ }^{5}$ Klinikum der Universität München, Ludwig- \\ Maximilians-University Munich, Munich, Germany
}

The most frequent complication after cardiac surgery is acute kidney injury (AKI). Macrophage migration inhibitory factor (MIF), a stress-regulating cytokine with proinflammatory properties, was previously shown to be cardioprotective in ischemia-reperfusion injury of the myocardium, but its role in the pathogenesis of AKI was unclear.

We have collected serum and urine samples from 60 patients scheduled for elective conventional cardiac surgery with the use of cardiopulmonary bypass and quantified levels of MIF by ELISA. We found that in patients, cardiac surgery triggered a significant increase in MIF serum concentrations. This increase was inversely and significantly correlated with urinary levels of kidney tubular cell injury marker, neutrophil gelatinase associated lipocalin (NGAL) indicating a possible protective role of MIF. Patients with high circulating MIF levels $12 \mathrm{~h}$ after surgery also had a significantly reduced risk for the development of AKI. We further analyzed serum of patients undergoing tumor enucleation, who were exposed to renal hypoxia by cross-clamping the renal artery vs. no hypoxia. Renal ischemia induced MIF release into the bloodstream and the duration of ischemia positively correlated with circulating MIF. To address the functional role of MIF, we used two different murine models of AKI induced by rhabdomyolysis for $24 \mathrm{~h}$ and unilateral or bilateral ischemia-reperfusion injury (I/R) for 6 or $24 \mathrm{~h}$. Compared to wild-type mice, Mif-deficient mice showed a significantly aggravated renal failure due to increased tubular cell injury in both models and at all times analyzed. This was associated with increased extent of programmed cell death, i.e. apoptosis, necroptosis and ferroptosis accompanied by enhanced oxidative stress. Therapeutic administration of recombinant MIF 30 minutes before and $6 \mathrm{~h}$ after renal ischemia-reperfusion injury in mice reduced the extent of tubular injury and tubular cell death, accompanied by improved kidney function. Treatment of primary murine tubular epithelial cells in vitro with recombinant MIF significantly reduced cell death and oxidative stress in the setting of hypoxia. Our data provide first evidence of a renoprotective role of MIF in AKI after cardiac surgery, likely by reducing regulated necrosis and mitigating oxidative stress in renal tubular epithelial cells.

\section{4 \\ The Inhibition of the WNK1 Kinase Worsens the Outcome of Ischemia/Reperfusion-Induced AKI}

Aïssata Niasse, Sandrine Placier, Samy Hamadache, Juliette Hadchouel

INSERM UMR_S1155, Hôpital Tenon, Paris, France

The With No lysine (K) serine-threonine kinase 1 (WNK1) is a key regulator of cellular homeostasis by tuning the activity of the family of cation-chloride co-transporters (SLC12A) following changes in intracellular chloride concentration and osmolarity. The impaired regulation of WNK1 could therefore affect the function of many tissues. This pathway has been extensively investigated in the distal nephron, where we showed that WNK1 activation leads to hypertension. However, the distal nephron is only a minor site of WNK1 expression in the kidney and several lines of evidence suggest that it could play an important role in others renal pathological conditions, particularly during Acute Kidney Injury (AKI). In vitro and in vivo studies showed that WNK1 plays a crucial role in the endothelium. Global as well as endothelial-specific disruptions of WNK1 result in angiogenic and cardiovascular defects and the death of WNK1-/- embryos around embryonic day 11. In HUVEC cells, WNK1 is required for endothelial cell proliferation, chemotaxis and migration. In addition, WNK1 participates in the regulation of vasoconstriction. We indeed showed that a $50 \%$ decrease in WNK1 expression in mice reduces the vasoconstriction of resistance arteries in response to pharmacological agents. Interestingly, the activation of the mineralocorticoid receptor (MR) in the arteries produces the reverse phenotype, with an increase in vasoconstriction. These results suggest that WNK1 may act downstream of aldosterone in the arteries, as is the case in the distal nephron. Since MR inhibition is beneficial in renal failure, we hypothesized that WNK1 inhibition could have a similar effect. Surprisingly, our recent data show the exact opposite. We tested the consequences of WNK1 knock-down in AKI provoked by ischemiareperfusion. The model we used is an heterozygous inactivation of WNK1 (WNK1+/- mice), in which WNK1 mRNA expression is decreased by $50 \%$ since WNK1-/- embryos die in 
utero. We observed that the partial inhibition of WNK1 worsens AKI provoked by a 30 minclamping of the renal artery followed by a 24 h-reperfusion. The tubular lesions are more severe in WNK1+/- mice than in WNK1+/+ animals after ischemia, as shown by an increased level of expression of Kidney Injury Molecule- 1 and Interleukin 6 . The tubular damages could be due to an aggravated endothelial dysfunction or an alteration of the survival of the proximal tubular cells, since WNK1 knock-down impairs cell division and survival in vitro. Further studies are required to define the mechanisms underlying tubular alterations in $\mathrm{WNK} 1+$ /animals after renal ischemia.

\section{5 \\ Dipeptidyl Peptidase-4 Inhibitor Teneligliptin Accelerates Recovery from Cisplatin Induced AKI by Promoting the Proliferation of Proximal Tubular Epithelial Cells}

Takamasa Iwakura, Hans-Joachim Anders

Division of Nephrology, Medizinische Klinik und Poliklinik IV, Klinikum der Universität, Munich, Germany

Dipeptidyl peptidase-4 (DPP-4) inhibitors can have renoprotective effects in some acute kidney injury (AKI) models, but the mechanisms-of-action and their role in tubule recovery upon AKI remains speculative. We hypothesized that the DPP-4 inhibitor teneligliptin can accelerate tubular re-epithelialization after cisplatin-induced AKI by triggering cell cycle entry and completion of mitosis, i.e. proliferation of tubular epithelial cells. Isolated primary tubular cells were screened with a drug library to identify potential candidates to accelerate proliferation by 3-(4,5-dimethylthiazol-2-yl)-2,5-diphenyltetrazolium bromide (MTT) assay. Proliferation of tubular cells was evaluated with cell cycle analysis and cell counting by flow cytometry. Rats were in-

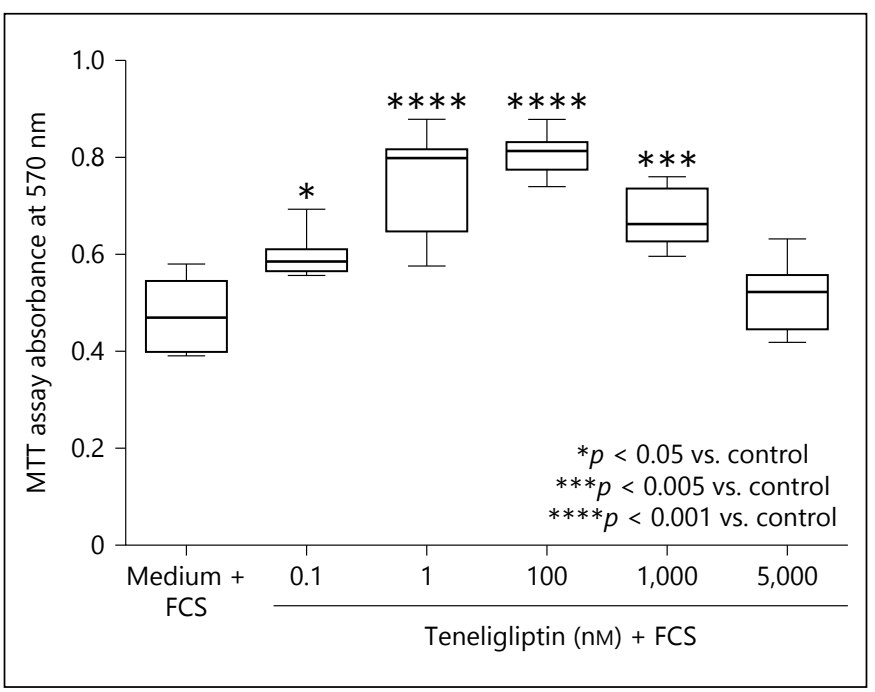

Fig. 1. (for Abstract 5). duced AKI by injecting $5 \mathrm{mg} / \mathrm{kg}$ of cisplatin intravenously. Oral administration of $10 \mathrm{mg} / \mathrm{kg}$ of teneligliptin, once a day, was started just before injecting cisplatin or from day 5 after cisplatin injection. In vitro, teneligliptin and its optimal dose were selected based on the MTT screening assay (Fig. 1). Teneligliptin promoted cell cycle entry into S/G2/M phase in primary tubular cells (Fig. 2), which increased cell number (Fig. 3). In vivo, the levels of serum Creatinine $(\mathrm{sCr}) / \mathrm{BUN}$ and cell death evaluated with the terminal uridine nick-end labeling (TUNEL) + cells peaked at day 5 after cisplatin injection. The levels decreased at day 10, comparing to that of day 5; however, the increased levels sustained until day 14. Pretreatment of teneligliptin prevented the elevation of sCr/BUN level and decreased TUNEL+ cells at day 5. Post treatment of teneligliptin from day 5 decreased BUN level (Fig. 4), Kidney Injury Molecule-1 (Kim-1) expression (Fig. 5) and col-

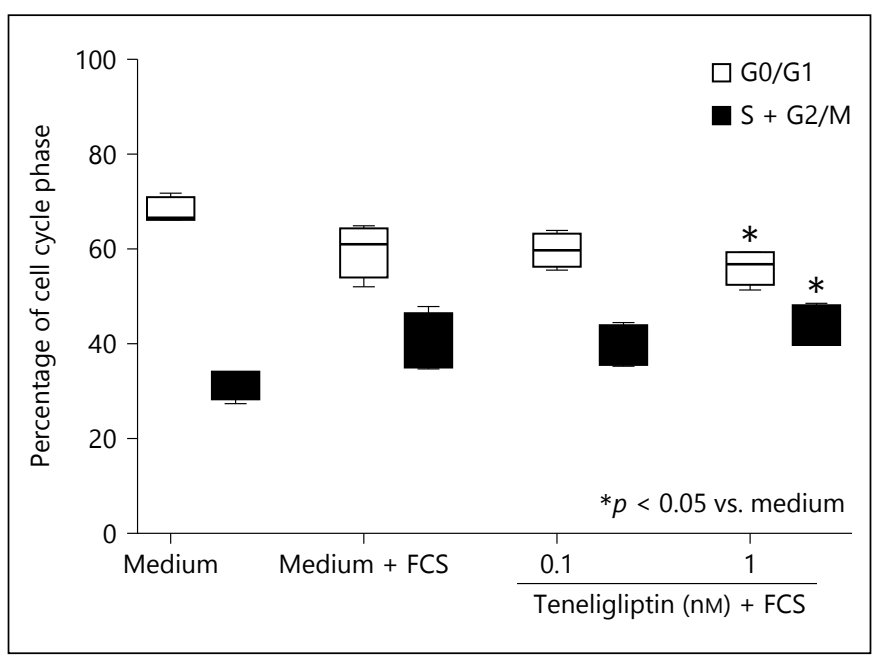

Fig. 2. (for Abstract 5).

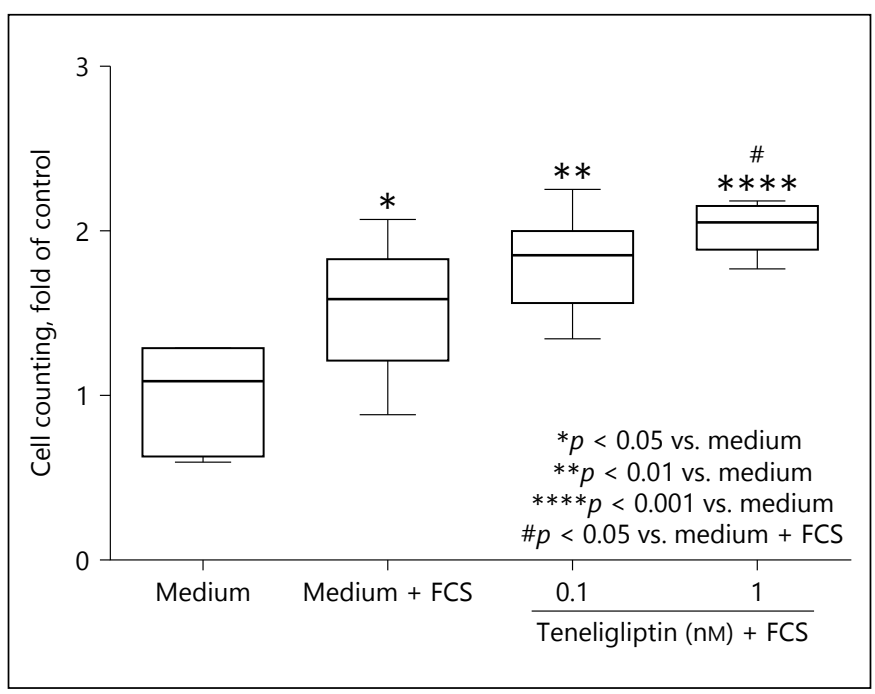

Fig. 3. (for Abstract 5). 


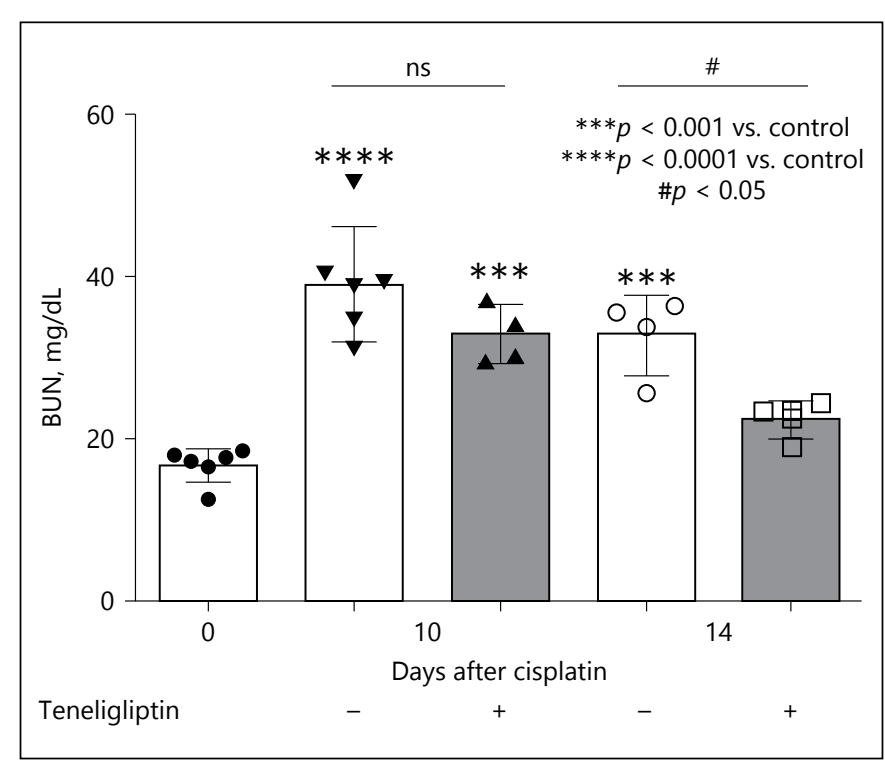

Fig. 4. (for Abstract 5).

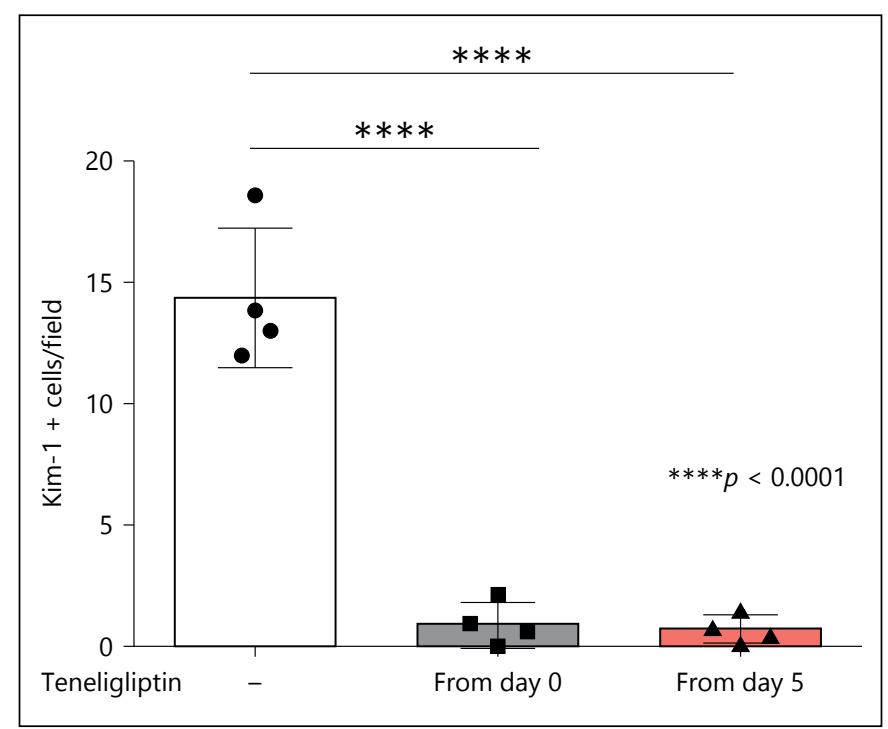

Fig. 5. (for Abstract 5).

lagen deposition (Fig. 6) at day 14. The DPP-4 inhibitor teneligliptin accelerates recovery after toxic AKI by facilitating the proliferation of tubular cells.

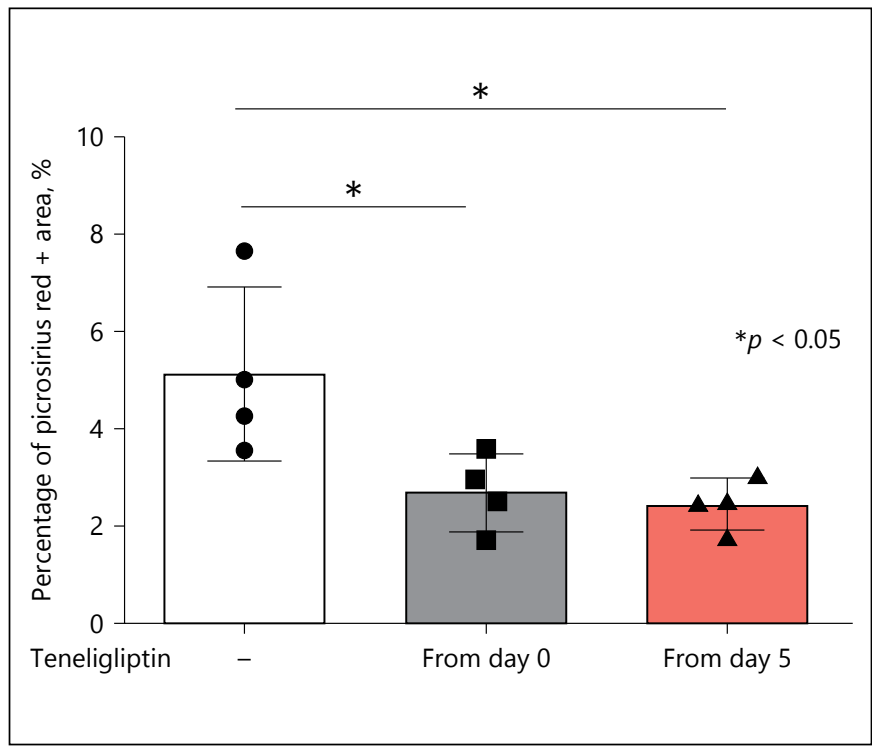

Fig. 6. (for Abstract 5).

\section{6}

\section{A Small Molecule Compound Library Screening Approach to Identify Drugs Targeting Tubular Cell Regeneration in Man and Mouse}

J.A. Marschner ${ }^{1}$, T. Iwakura ${ }^{1}$, F. Kundert ${ }^{1}$, L. Platen ${ }^{1}$, Z. Zhao', P. Romagnani ${ }^{2}$, H.J. Anders ${ }^{1}$

${ }^{1}$ Department of Clinical Biochemistry, Medical Clinic University of Munich, Munich, Germany; ${ }^{2}$ Department of Clinical and Experimental Biomedical Sciences, University of Florence, Italy

Currently no treatments are approved to accelerate tubule regeneration upon acute kidney njury (AKI). The discovery of scattered progenitor cells among the tubular epithelial cells prompted us to identify potential drug targets to accelerate the kidney's intrinsic capacity for tubule regeneration upon AKI. Our goal is to identify such molecules in a most efficient fashion based on ex vivo and in vitro techniques that delivers a profound rationale for translational in vivo experiments. A stem cell-focused molecular library of 155 small molecules was tested for induction of metabolic activity in primary murine proximal tubular cells and human tubular progenitor cells from different donors, both being relevant target cells under in vivo conditions of tubular cell regeneration. A heat-map assisted analysis identified a subset of 22 possible candidate compounds that were further tested in detail for dose-dependent effects on metabolic activity. For the most promising compounds RKI (ROCK-inhibitor) and SB (ALK5SMAD2/3-inhibitor) we confirmed an induction of hyperplasia rather than hyperthrophy by coulter counting under different culture conditions. Their respective capacities to induce wound healing and regeneration were studied by using electric cellsubstrate impedance sensing, cytotoxicity and viability assays as well as cell proliferation upon injury with histones, a danger-associated mol- 


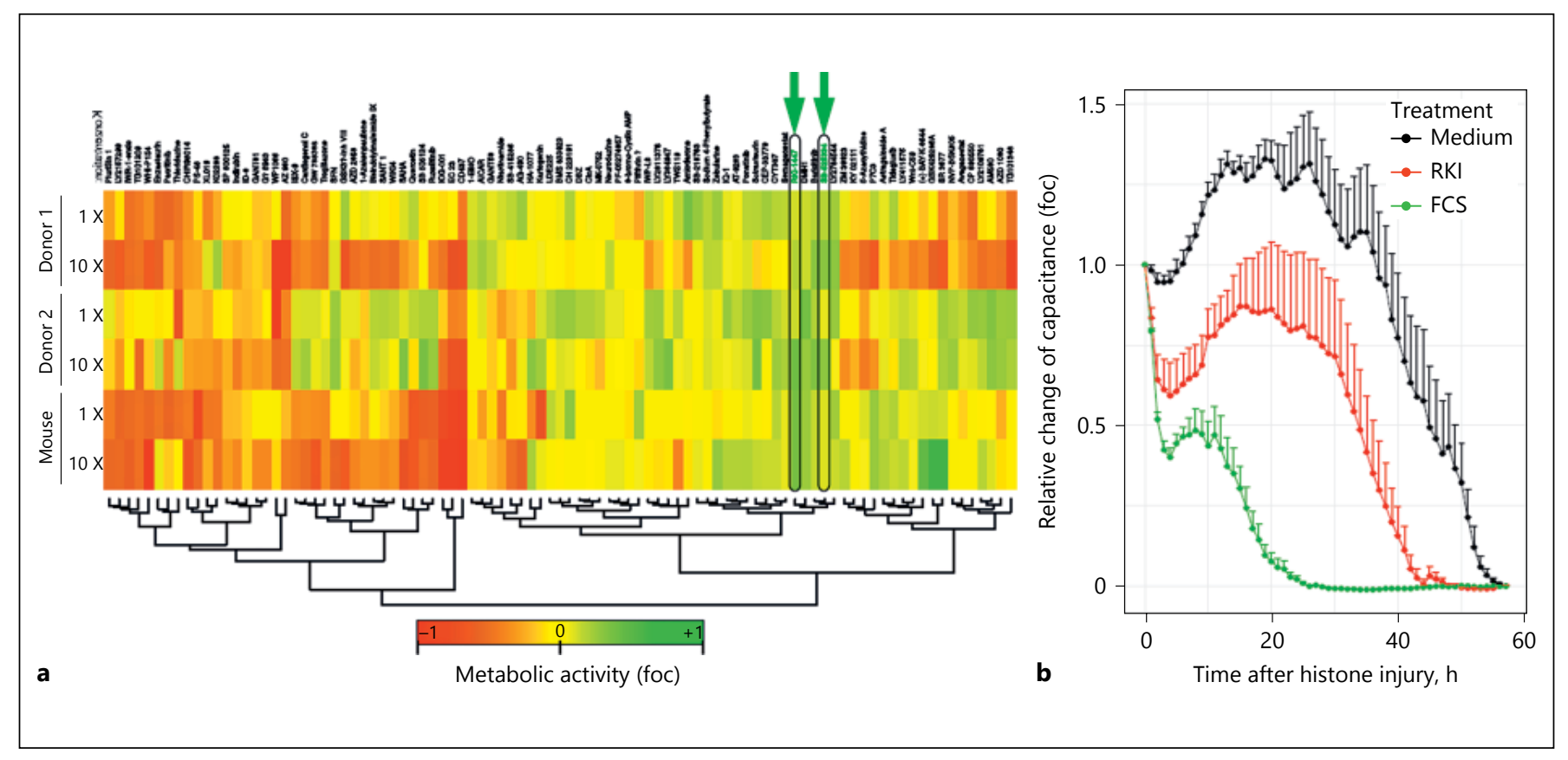

Fig. 1. a Preselection of candidate compounds from a stem cellfocused library of small molecules by screening for metabolic activity and cell counting in human renal progenitor and murine primary tubular cells. b Wound closure and regeneration capaci-

ecule playing a predominant role in different forms of AKI. RKI proved to be a powerful small molecule that drives wound healing and regeneration of murine and human tubular cells. We are currently studying its performance in a murine AKI model of renal ischemia reperfusion.

\section{7 \\ Renal Tubular GB3 is a Receptor for Proteins and Xenobiotics}

Ivan Morace, Giuseppina Federico, Ina Schießl, Elisabeth Gröne, Ludger Johannes, Hermann-Josef Gröne

German Cancer Research Center, Department Cellular and Molecular Pathology, Heidelberg, Germany

In order to elucidate the physiologic function of renal globotriaosylceramide (Gb3/CD77), the Shigatoxin (STx) receptor, we have analyzed renal function in GB3 synthase k.o. (Gb3S-/-) mice. These mice displayed an increased renal albumin excretion compared to wild type (WT), which was even more pronounced in mice fed with a high fat diet for 4 weeks. Gb3 localized both at the brush border and within vesicular structures in WT proximal tubules (PT), co-localizing partially with megalin and cubilin. In mouse models of acute kidney injury caused by myoglobinuria and aminoglycoside, GB3S-/- mice showed a preserved renal function and morphology compared to WT; this was due to a lower uptake of toxic myoglobin and gentamycin ties of RKI after histone-induced injury studied by electric cellsubstrate impedance sensing as compared to medium- and FCScontrol (for Abstract 6).

by PTs. Pharmacologic inhibition of glucosylceramide-based glycosphingolipids in WT mice corroborated the results of genetically Gb3-deficient mice. In conclusion, we have shown that Gb3 is required for optimal PT reabsorption of filtered proteins and xenobiotics.

\section{8 \\ Molecular Characterization of Nephrosphere- Derived PKH ${ }^{\text {high }} /$ CD133+/CD24- Stem-Like Cells and their Use in Repopulation of Decellularized Kidney Scaffolds}

Silvia Bombelli ${ }^{1}$, Chiara Meregalli ${ }^{1}$, Maddalena Bolognesi ${ }^{1}$, Grazisa Rossetti ${ }^{2}$, Valeria Ranzani ${ }^{2}$, Barbara Torsello ${ }^{1}$, Sofia De Marco ${ }^{1}$, Massimiliano Pagani ${ }^{2}$, Giorgio Cattoretti ${ }^{1}$, Paolo Viganò ${ }^{3}$, Cristina Bianchi ${ }^{1}$, Roberto Perego ${ }^{1}$

${ }^{1}$ School of Medicine and Surgery, Milano-Bicocca University, Monza, Italy; ${ }^{2}$ Istituto Nazionale Genetica Molecolare "Romeo ed Enrica Invernizzi", Milano, Italy; ${ }^{3}$ Urology Unit, Bassini ICP Hospital, Milano, Italy

Kidney regeneration as a therapeutic option for chronic kidney diseases is particularly difficult because of the anatomical complexity of the organ. As a tool for renal reconstitution, to date a valuable approach seems to be the recellularization of decellularized scaffolds. Many attempts of decellularization and recel- 
lularization have been performed in different species and with different cell types, even if the ultimate cell type, able to reconstitute various portions of renal architecture, is still not so well understood. We obtained nephrospheres (NS) from adult human renal tissue that could repopulate slices of human renal scaffolds both in tubular and in vascular components. Moreover, we previously identified cells with stem properties as a subpopulation within the NS, as $\mathrm{PKH}^{\text {high }} / \mathrm{CD} 133+/ \mathrm{CD} 24$ - cells (RSC) able to self-renew and to differentiate along different lineages. Therefore, we now aim to prove the ability of $\mathrm{PKH}^{\text {high }}$ cells alone to repopulate decellularized renal slices. We thus cultured $\mathrm{PKH}^{\text {high }}$ cells on decellularized kidney slices for 30 days and we showed, for the first time, that $\mathrm{PKH}^{\text {high }}$ cells were able to repopulate both tubular and vascular segments of renal scaffold differentiating in epithelial and endothelial lineages. Of note, our $\mathrm{PKH}^{\text {high }}$ cells, that do not express endothelial markers, can generate secondary nephrospheres containing cells with an endothelial phenotype, as well as endothelial-like structures on decellulalrized scaffolds. We also aim to find the molecular signature of RSC subpopulation to better disclose the phenotype hidden behind $\mathrm{PKH}^{\text {high }}$ functional status. Transcriptomic analysis of RSC compared with their $\mathrm{PKH}^{\text {low/neg }}$ progeny was performed and differentially expressed genes (DEG) were evidenced. Bioinformatic Gene Set Enrichment Analysis suggested a renal early status of differentiation of our RSC, different from embryonic stem cells. We finally obtained a RSC signature, and three different gene products were selected as potential markers and validated by immunofluorescence on $\mathrm{PKH}^{\text {high }}$ sorted cells. Single cells were obtained from adult human renal tissue for testing the potential stem markers for FACS analysis. We identified cells expressing the selected markers, evidencing a specific phenotype in adult renal tissue. We are now going to isolate and better characterize these cells for their functional abilities. In conclusion, we highlighted the proximal and distal tubular epithelial and endothelial differentiative and regenerative abilities of $\mathrm{PKH}^{\text {high }}$ cells. Moreover, we showed that $\mathrm{PKH}^{\text {high }}$ cells, completely lacking any endothelial marker, were able to give rise to endothelial-like structures. Finally, the obtained RSC signature would open the possibility for the direct identification and, hopefully, isolation of adult renal stem-like cells from normal kidney tissue.

\section{9 \\ PDGFRa-Deletion in FoxD1+-Mesenchymal Cells Markedly Reduces Renal Fibrosis in the Mouse Ischemia/Reperfusion Model}

Ina V. Martin ${ }^{1}$, Maria Mattiotti ${ }^{2}$, Ute Raffetseder ${ }^{1}$, Jürgen Floege ${ }^{1}$, Tammo Ostendorf

${ }^{1}$ Division of Nephrology, Med. Clinic II, RWTH Aachen

University, Aachen, Germany; ${ }^{2}$ Department of Nephrology,

Dialysis and Transplantation, Fondazione IRCCS Policlinico San

Matteo, Pavia, Italy

Background: The Platelet-Derived Growth Factor Receptoralpha (PDGFR $\alpha$ ) is constitutively expressed in the kidney and strongly upregulated during renal fibrosis. Its ligand PDGF-C has been shown to have strong pro-fibrotic properties. The PDGFRa- expressing FoxD1+ pericytes and perivascular fibroblasts transdifferentiate to extracellular matrix-secreting myofibroblasts in settings of kidney fibrosis. We now investigated the specific role of FoxD1+ mesenchymal cell-expressed PDGFR $\alpha$ in experimental renal fibrosis.

Methods: To this aim we first generated mice with PDGFR $\alpha$ deletion specifically in FoxD1+ cells. Kidneys of healthy 8-weekold PDGFRafl/fl FoxD1cre/+-mice (KO) and wildtype (WT)littermates were analyzed. In addition, 10-week-old male KO-mice $(n=6)$ and WT-littermates $(n=7)$ underwent unilateral ischemia/ reperfusion injury (I/R) and kidneys were analyzed at day 21 following I/R. Main tissue analyses were done by real-time RT-PCR and (immuno)histochemistry.

Results: The mesenchymal cell-specific PDGFRa KO-mice were viable, although born at only half the expected Mendelian frequency. They showed a slightly reduced bodyweight and kidney/body weight-ratio compared to their WT-littermates. In 8 -week-old male mice renal Pdgfra expression was reduced by $86 \%$ and Pdgfrb expression by $36 \%$ in KO compared to WT. Immunhistochemical stainings showed a $92 \%$ decrease in cortical PDGFRa-positivity in KO- compared to WT-mice. The gene expression of PDGF-ligands was unaltered in KO-mice, whereas expression of angiogenesis-relevant genes encoding VEGFR1, VEGFR2, ANGPT2 and MECA32 was significantly reduced. In the 21-day-I/R-model fibrotic gene expression (Acta2, Col1A1, Co$13 \mathrm{~A} 1, \mathrm{FN})$ was strongly reduced by $73-80 \%$ in the KO mice. Cortical aSMA protein was decreased by $50 \%$ in $\mathrm{KO}$ - compared to WTmice, and collagen IV and collagen I levels were diminished by $36 \%$ and $25 \%$, respectively, as analyzed by immunohistochemistry. Finally, gene expression of chemokines CCL5 and CCL2 was downregulated 4-5-fold in the $\mathrm{KO}$ mice compared to their WT counterparts, and macrophages were slightly decreased.

Conclusions: Signaling via renal mesenchymal PDGFRa is central for the development of tubulointerstitial fibrosis following ischemia/reperfusion injury and may also impact on renal angiogenesis.

\section{0 \\ The SEMA3C/NRP1 Signalling Pathway in the Pathogenesis of Kidney Disease}

Gwanyu Ye ${ }^{1}$, Brigitte Surin ${ }^{1}$, Sandrine Placier ${ }^{1}$, Christos Chadjichristos ${ }^{1}$, Jean-Claude Dussaule ${ }^{1}$, Peter Scambler ${ }^{2}$, Christos Chatziantoniou ${ }^{1}$, Amelie Calmont ${ }^{1}$

${ }^{1}$ Kidney Research Centre, INSERM UMR S 1155, Tenon Hospital, 4 Rue de la Chine, Paris, France; ${ }^{2}$ Developmental Biology and Cancer Department, UCL-Institute of Child Health, 30 Guilford Street, London WC1N 1EH, UK

Semaphorins are guidance proteins which influence cellular morphology and function and play important roles in organogenesis and disease. The secreted glycoprotein SEMA3C plays deleterious roles in pathological conditions and its receptor Neuropilin1 (NRP1) promotes fibrosis progression in the liver.

In the kidney, the SEMA3C/NRP1 signalling axis plays an essential role in chronic kidney disease progression. We found that SEMA3C was not only an early marker of renal damage in both 
humans and mice but also an active player in disease progression. Indeed, the severity of renal lesions was reduced in Sema3C conditional mutants after kidney injury. In addition, we also detected the presence of SEMA3C in the urine of renal disease patients prior to the onset of albuminuria.

SEMA3C is also highly expressed upon acute kidney injury (AKI) and genetic inactivation of Sema3C protects mice from folic acid-induced AKI, improves tissue histology, reduces neutrophil infiltration and prevents epithelial cell apoptosis.

By acting on NRP1-positive endothelial cells, our data suggest that SEMA3C-driven pathways play deleterious roles in microvasculature rearrangements (permeability and capillary rarefaction) and that early functional alteration of peritubular capillaries could initiate/participate in fibrosis production.

\section{1 \\ Investigating the Endocycle-Mediated Response in Human Renal Tubular Epithelial Cells}

\author{
Letizia De Chiara ${ }^{1,3}$, Elena Lazzeri ${ }^{1,3}$, Maria Lucia Angelotti ${ }^{1,3}$, \\ Carolina Conte ${ }^{1,3}$, Anna Peired ${ }^{1,3}$, Giulia Antonelli $i^{1,3}$, \\ Maria Elena Melica ${ }^{1,3}$, Marco Allinovi ${ }^{1,2,3}$, Benedetta Mazzinghi ${ }^{1,3}$, \\ Laura Lasagni, ${ }^{1,3}$, Paola Romagnani ${ }^{1,2,3}$ \\ ${ }^{1}$ Department of Biomedical Experimental and Clinical \\ Sciences "Mario Serio", University of Florence, Florence, Italy; \\ ${ }^{2}$ Nephrology and Dialysis Unit, Meyer Children's University \\ Hospital, Florence, Italy; ${ }^{3}$ Excellence Center DENOTHE, \\ University of Florence, Florence, Italy
}

Acute kidney injury (AKI) is a global public health concern impacting $\sim 13.3$ million patients per year. If not lethal in the acute phase, AKI is considered reversible. However, even mild AKI episodes carry substantial risk of developing subsequent chronic kidney disease (CKD). The pathophysiological basis for this phenomenon remains unclear.

Functional recovery from AKI was traditionally attributed to the regenerative potential of tubular epithelial cells (TECs) which are believed to re-enter the cell cycle and repair the damage. Nevertheless, our recent data provide evidence that an endocycle-mediated response of remnant TECs may represent a critical mechanism of response to AKI. Endocycles are cell cycle variants consisting of $G$ and $S$ phases alone that repeatedly proceed without cytokinesis. Crucially, the role of endocycle in repair of human tissues is mostly unknown and totally unexplored in the kidney.

Aim of the Study: Evaluate the presence of abnormal cell cycles in human renal cells and identify possible genes/pathways that regulate TEC-endocycle.

Methods and Results: To effectively analyze the endocycle of human RPTECs (renal proximal tubular epithelial cells) and taking advantage of the pRetroX-G1-mCherry/SG2M-Cyan vectors (collectively defined as the FUCCI retroviruses) available in the lab, I recently generated RPTEC/FUCCI cells. Specifically, the expression of the fluorescent protein mCherry (red) is driven in the nuclei of cells in G1 phase and of AmCyan (cyan) in the nuclei of cells in S/G2/M phase. Intriguingly, my preliminary data show that RPTEC/FUCCI cells undergo endocycles in culture, defined as cells in G1 (mCherry ${ }^{+}$) with a DNA content twice or higher than regular G1 cells. Importantly, the number of endocycling cells increases when the cells are exposed to a damage (i.e. Aristolochic Acid or TGF $\beta$ treatment for $48 \mathrm{~h}$ ) and not when they are exposed to a compound that blocks the cells in G2 (RO-3306) indicating that what we observed it is not a simple cell cycle arrest, as previously suggested. Remarkably, endocycle-mediated response is prevented when the human renal cells are treated with a pathwayspecific inhibitor. Finally, evidence of endocycle-mediated response has been found in kidney biopsies of CKD patients.

Conclusions: Collectively, these results suggest that human RPTECs are able to undergo endocyle-mediated response after a damage and that endocycle is driven by a pathway prominently involved in cell proliferation. Furthermore, these important findings also open up to the possibility of employing endocycling cells as a prognostic marker in CKD patients.

12

(withdrawn on April 25, 2018) 


\section{3 \\ The Cold Shock Domain Protein YB-1 Plays a Critical Role in Sterile Tubulointerstitial Nephritis}

Anja Bernhardt, Saskia Jerchel, Sabine Brandt, Katherina Wolters, Maria Sounidaki, Jonathan A. Lindquist, Peter R. Mertens

Department of Nephrology and Hypertension, Diabetes and Endocrinology, Otto-von-Guericke-University Magdeburg, Germany

The Y-box binding protein-1 (YB-1) belongs to the family of highly conserved cold shock domain proteins. By its ability to bind single stranded DNA or RNA, YB-1 influences the transcriptional and translational processing of many genes e.g. pro-inflammatory genes like RANTES/CCL5 and pro-fibrotic genes like TGF- $\beta$. Since the conventional homozygous knockout of $\mathrm{Ybx} 1$ is embryonically lethal, we generated a Rosa26CreERT2 inducible Ybx1 knockout mouse to investigate the influence of the whole body knockout of YB-1 on cell function and disease development. The disease model of unilateral ureteral obstruction (UUO) was applied in animals, which results in acute sterile tubulointerstitial damage and progressive kidney fibrosis. The Cre recombinase was activated by oral gavage with a single dose of tamoxifen. Our data revealed a significant reduction of YB-1 protein expression in blood (45\%), spleen (69\%), bone marrow (61\%), and kidney (30\%) cells 28 days after tamoxifen application. After disease induction wildtype mice showed a strong infiltration of immune cells to the site of damage whereas KO mice showed significantly lower infiltration of leukocytes compared to the wildtype (61\%). Using Sirius Red staining we could quantify $40 \%$ lower collagen deposition in YB1 ${ }^{\triangle \text { RosaERT2 }}$ knockout mice 14 days after UUO. To further our understanding of inflammation and fibrosis we analyzed the inflammatory micromilieu (e.g. TGF- $\beta$, RANTES) in UUO kidneys. All values were lowered in knockout animals, demonstrating the role of YB-1 in regulating proinflammatory responses. Moreover, ligation of the left ureter leads to the congestion of urine in the kidney and increased intrarenal pressure. Using Periodic acidSchiff (PAS) staining in order to visualized the extensive dilation of collecting ducts (cd), distal tubulus (dt) and proximal tubulus (pt). Following UUO tubular cell damage and apoptosis ensues; the number of tubular epithelial cells that are attached to the basement membranes over the whole nephron is significantly enhanced in YB-1 $1^{\triangle \text { RosaERT2 }}$ knockout mice compare to wildtype, indicating less apoptotic activity when $\mathrm{YB}-1$ is genetically ablated. In summary, our data demonstrate that a single induction of the Cre recombinase is sufficient to generate a knockout of YB-1. These animals are viable and exhibit significantly reduced immune cell infiltration after UUO. Furthermore, the collagen deposition after disease induction was significantly reduced, similar to findings with heterozygote YB-1 knockout animals [1]. Thus, in contrast to our previous results obtained following ischemia/reperfusion damage where YB-1 acts protective on tubular cell survival [2], YB-1 depletion is of benefit in the sterile tubulointerstitial nephritis model with impaired immune response and collagen deposition.

\section{References}

1 Wang J, Gibbert L, Djudjaj S, Alidousty C, Rauen T, Kunter U, Rembiak A, Enders D, Jankowski V, Braun GS, Floege J, Ostendorf T, Raffetseder U: Therapeutic nuclear shuttling of YB-1 reduces renal damage and fibrosis. Kidney Int 2016;90(6):1226-1237.

2 Dong W, Wang H, Shahzad K, Bock F, Al-Dabet MM, Ranjan S, Wolter J, Kohli S, Hoffmann J, Dhople VM, Zhu C, Lindquist JA, Esmon CT, Gröne E, Gröne HJ, Madhusudhan T, Mertens PR, Schlüter D, Isermann B: Activated Protein C Ameliorates Renal Ischemia-Reperfusion Injury by Restricting Y-Box Binding Protein-1 Ubiquitination. J Am Soc Nephrol 2015;26(11):2789-2799.

\section{4}

\section{YB-1 Mediated CXCL-1 Expression Decides on Kidney Damage in Liver Fibrosis}

Daniela Hermert ${ }^{1}$, Ina V. Martin ${ }^{1}$, Kathy Reiss ${ }^{2}$, Daniel Breitkopf ${ }^{1}$, Jürgen Floege ${ }^{1}$, Tammo Ostendorf', Ralf Weiskirchen ${ }^{3}$, Ute Raffetseder ${ }^{1}$

${ }^{1}$ Department of Clinical Immunology and Nephrology, RWTH Aachen University, Germany; ${ }^{2}$ Institute of Pharmacology and Toxicology, Medical Faculty, RWTH Aachen University, Germany; ${ }^{3}$ Institute of Molecular Pathobiochemistry, Experimental Gene Therapy and Clinical Chemistry, RWTH Aachen University; Germany

The Y-box binding protein-1 (YB-1) exerts pleiotropic functions in gene transcription and in translation, e.g. of fibrosis-related genes. Herein, we analysed the impact of half-maximal expression of YB-1 in a model of liver fibrosis, monitored the renal injury induced by liver dysfunction and identified a mediator important in this organ-cross-talk.

The impact of complete obstruction of the common bile duct (BDL) was analysed in liver and kidneys in $\mathrm{Ybl}^{+/-}$mice and their wildtype (WT) littermates 14 days after disease induction. Parameters of inflammation/fibrosis were determined by immunohistochemistry and qRT-PCR in liver and kidney tissues. Furthermore, YB-1-mediated expression of the pro-inflammatory chemokine CXCL1 was analysed in gel shifts, qRT-PCR and gene reporter assays in cell lines and primary renal and hepatic cells. In vivo, pharmacological inhibition of the CXCL1 receptor CXCR2 by SB225002 was tested in the early phase of the BDL model that is characterized by granulocyte infiltration and the onset of liver/kidney damage.

Following BDL, hepatic serum parameters and hepatic expression of ECM proteins such as type I collagen (CollA) were significantly reduced in $\mathrm{Yb1}^{+/-}$compared to WT mice, whereas expression of chemokine CXCL1 was augmented in $\mathrm{Yb1}^{+/-}$animals. Via depletion-, overexpression-, reporter gene- and gel shift-assays, YB-1 was identified as a potent transcriptional repressor of Cxcl1 gene expression. Enhanced systemic CXCL1 content in $\mathrm{Yb}^{+/-}$mice exhibited pronounced effects on kidneys following $\mathrm{BDL}$ compared to their WT littermates that were monitored via parameters of tubular damage and immune cell infiltration. Con- 
sequently, pharmacological inhibition of the CXCL1 receptor CXCR2 significantly reduced early systemic effects on the kidneys following BDL.

This analysis provides direct evidence that YB-1 crucially contributes to hepatic fibrosis and concurrently modulates organ crosstalk between liver and kidney through transcriptional repression of chemokine CXCL1 expression.

\section{5 \\ Distinct Roles of Receptor Notch3 Activation on Immune Cells or Tubular Cells for Tubulointerstitial Kidney Injury}

Sabine Brandt ${ }^{1}$, Tobias Ballhause ${ }^{1,2}$, Florian Heidel ${ }^{3,4}$, Anja Bernhardt ${ }^{1}$, Alexander Fehr ${ }^{1}$, Yan Fu ${ }^{5}$, Andreas Müller ${ }^{5}$, Lars Philipsen ${ }^{5}$, Annika Becker ${ }^{1}$, Peter R. Mertens ${ }^{1}$

${ }^{1}$ Clinic of Nephrology and Hypertension, Diabetes and Endocrinology, Otto-von-Guericke University Magdeburg, Germany; ${ }^{2}$ current address:Clinic for accident, Hand and Reconstructive Surgery, University Hospital HamburgEppendorf, Germany; ${ }^{3}$ Department of Hematology and Oncology, Otto-von-Guericke University Magdeburg, Germany ${ }^{4}$ Department of Haematology and Medical Oncology, Friedrich Schiller University, Jena, Germany; ${ }^{5}$ Institute of Molecular and Clinical Immunology, Otto-von-Guericke University Magdeburg, Germany

In most glomerular kidney diseases 'mediators' within the primary urine incite and perpetuate the inflammatory response within tubular epithelial cells and the tubulointerstitium, which is accompanied by inflammatory cell recruitment. Infiltrating monocytes release mediators and activate cells. Y-box (YB) binding protein-1 is present in urine samples of glomerulonephritis patients and interacts with extracellular residues of receptor Notch-3 on tubular cells. In vivo analyses revealed enhanced expression and activation of receptor Notch-3 in tubular cells following unilateral ureteral obstruction (UUO) or in podocytes following injection of nephrotoxic serum. Receptor Notch-3 knockout mice are protected in both injury models as we observed less inflammation and fibrosis. To investigate the cell-specific functions of Notch-3, we generated chimeric mice lacking Notch-3 expression in bone marrow-derived or kidney resident cells using bone marrow transplantation. UUO was induced and animals analyzed after 5 and 14 days. Chimeras are viable and Notch-3-deficient cells show no defect in homing. Immunohistochemistry and flow cytometry revealed a Notch-3 receptor dependent infiltration of immune cells within inflamed tissue. The number of infiltrating macrophages in Notch-3-deficient bone marrow chimeras (Notch-3 $3^{\mathrm{ko} / \mathrm{wt}}$ ) following disease induction is significant decreased. Transplantation of wildtype bone marrow into receptor Notch-3 knockout mice (Notch- $3^{\text {wt } / \mathrm{ko}}$ ) results in an equal number of infiltrating immune cells compared to wild-type mice. Since the number of immune cells within the circulation does not differ between the groups, we suggest that Notch-3-deficient immune cells have a transendothelial migration or adhesion defect within the tissue. To analyze this, adoptive transfer experiments were established. Labeled wild-type bone marrow-derived immune cells were efficiently recruited, whereas the number of receptor Notch-3 knockout cells is markedly reduced. This correlates with the composition of the inflammatory milieu. Less infiltration of immune cells means less inflammatory cytokines and vice versa. The degree of fibrosis (analyzed by extracellular matrix deposition, SMA, and collagen expression) revealed no differences in Notch-3-deficient bone marrow chimeras (Notch-3 $3^{\mathrm{ko} / \mathrm{wt}}$ ) compare to wild-type, whereas in wild-type bone marrow chimeras with Notch3-deficient recipients (Notch$3^{\mathrm{wt} / \mathrm{ko}}$ ) we observed less fibrosis. Here, we described distinct roles of receptor Notch-3 in immune cells versus tissue resident cells. Expression of receptor Notch-3 on immune cells is required for leukocyte adhesion and transendothelial migration whereas receptor Notch-3 on resident cells appears to control fibrosis. This hypothesis is supported by several studies that describe the role of Notch receptors and their ligands in leukocyte adhesion to the endothelial by upregulating and increasing the binding affinity of adhesion molecules, including $\beta 1$ integrin and CD44.

\section{6 \\ The Dual Role of Arg Tyrosine Kinase in Tubulointerstitial Fibrosis}

B. Torsello, S. De Marco, S. Bombelli, V. Cassina, E. Chisci, C. Bianchi, R. Perego

School of Medicine and Surgery, University of Milano-Bicocca, Monza, Italy

Diabetic nephropathy is a complication occurring in 35\% of patients affected by diabetes mellitus and is the leading cause of end-stage renal disease in the developed world. Chronic exposure to elevated blood glucose concentration contributes to the tubulointerstitial changes, characterized by thickening of tubular basement membrane, tubular atrophy and interstitial fibrosis. Several in vivo models show that interstitial pericytes and resident fibroblasts might be progenitors of matrix-producing myofibroblasts. Nevertheless, the involvement of tubular cells in interstitial fibrosis has been suggested by the finding that under in vitro high glucose conditions proximal tubular cells overproduce TGF- $\beta 1$, one of the most important fibrogenic cytokines involved in development of renal interstitial fibrosis. Recently, we demonstrated that in human primary tubular cell cultures exposed to highglucose (HG) the ROS-dependent downregulation of tyrosine kinase Arg induced TGF- $\beta 1$ overproduction. In addition, we showed a further TGF- $\beta 1$ increase after Arg silencing in HGtreated tubular cells. TGF- $\beta 1$ produced by HG-treated tubular cells activated NIH3T3 fibroblasts inducing proliferation and $\alpha$-sma expression via smad pathway. Notably, other authors demonstrated that TGF- $\beta 1$ is unable to activate murine embrionic fibroblasts (Mef) lacking both Abl and Arg. To investigate the specific role of Arg isoforms in fibroblast activation, we transfected 1ALCTL and 1BLCTL isoforms in Arg KO Mefs. By proliferation assay and PCNA staining, we showed that the Arg KO fibroblast proliferation was decreased as compared to wt cells, and 1BLCTL but not 1ALCTL transfection, partially rescued cell proliferation. Loss of Arg caused a strong decrease of $\alpha$-sma expression in Mefs that only 1BLCTL isoform was able to rescue to wt level. Arg KO decreased also the 
nuclear localisation of Yap, a marker of fibroblast activation and the transfection of 1ALCTL and 1BLCTL isoforms was able to increase the nuclear Yap fraction. Wound healing and collagencoated Boyden assays showed that the loss of Arg increased the migration and the invasion ability of Mefs and 1BLCTL Arg isoform transfection further increased this migration ability. Furthermore, we analysed the capability of the different Mefs to remodel the extracellular matrix. By contraction assay, we showed that Arg loss in Mefs strongly decreased their collagen I matrix contraction ability, while 1ALCTL and 1BLCTL isoform transfection was able to rescue the matrix contraction percentage to Mef wt level. Furthermore, we induced Mefs to produce and remodel their extracellular matrix by Ascorbic Acid treatment. By IF, we showed that Arg KO decreased Mef collagen I deposition and both Arg isoform transfection increased collagen deposition. In particular, 1ALCTL isoform induced more prominent collagen I bundles, while 1BLCTL isoform induced the deposition of collagen IV bundles thicker than those observed in Mefs wt. Thicker collagen fibres are described to be associated with an increase of ECM stiffness in fibrotic tissues. To asses this correlation, we measured the stiffness of Mef-producing matrices by Atomic Force Microscopy. We showed that ECM stiffness of Arg KO Mefs was decreased with respect to wt Mefs and only 1BLCTL transfection in KO Arg Mefs rescued ECM stiffness. In conclusion, Arg plays a dual role in tubularinterstitial fibrosis: i) it modulates TGF- $\beta 1$ secretion by HG-treated tubular cells, and ii) it is essential for Mef activation and the two Arg isoforms analysed seem to be differently involved in some aspects of myofibroblast activation.

\section{7 \\ The Effect of In Vitro Tacrolimus Exposure and Pharmacogenetic Variation on Multilevel CYP3A5, ABCB1 Expression and CTGF Production in Human Proximal Tubule Cells \\ Noel Knops ${ }^{1,2}$, Yasaman Ramazani $^{2}$, Bert P. van den Heuvel ${ }^{2,3}$, Elena Levtchenko ${ }^{1,2}$, Dirk J. Kuypers ${ }^{4}$ \\ ${ }^{1} U Z$ leuven, pediatric nephrology and solid organ transplantation, Leuven, Belgium; ${ }^{2} \mathrm{KU}$ Leuven, pediatric nephrology, Leuven, Belgium; ${ }^{3}$ Radboud university medical center, translational metabolic laboratory, Nijmegen, Netherlands; ${ }^{4} \mathrm{UZ}$ Leuven and KU Leuven, nephrology and renal transplantation, Leuven, Belgium}

Background: The interplay between CYP3A enzymes and the P-gp pump (ABCB1) is important for drug metabolism and toxicity. Genetic variation in CYP3A5 and $\mathrm{ABCB} 1$ are important in tacrolimus (Tac) nephrotoxicity. We show for the first time, the implications of Tac exposure in a novel human PTC model incorporating the genetic variation in CYP3A5 and ABCB1 on the function of these genes and the production of pro-fibrotic cytokines.

Methods: Eight clones of conditionally immortalized PTC (ciPTC) with different combinations of CYP3A5 (rs776746) and ABCB1(rs1045642) genotypes were incubated with vehicle, $50 \mathrm{ng} /$ $\mathrm{mL}$ and $300 \mathrm{ng} / \mathrm{mL}$ Tac for 24 and $72 \mathrm{~h}$. qRT-PCR and western blot were performed for CYP3A5, ABCB1 and CTGF expression. Functional CYP3A5 expression was assessed by differential mid- azolam (MDZ) hydroxylation using LC-MS and P-gp activity by calcein efflux.

Results: Baseline mRNA, protein and functional expression of CYP3A5 was higher in ciPTC with the ${ }^{*} 1$ versus $* 3 / * 3$ allele. Increasing Tac concentration resulted in decreasing 1'OH MDZ hydroxylation $(\mathrm{p}=0.01)$. Baseline mRNA and calcein-AM efflux was higher in ABCB1 3435 TT vs. CC/CTs (mean $\triangle \mathrm{Ct}$ ABCB1: 2.25 vs. 2.97 and $\Delta$ fluorescence: $23.4 \%$ vs. $45.3 \%$, respectively; $\mathrm{p}=0.001$ ). A progressive decrease in calcein efflux (50 ng/mL: 79.1\%; $300 \mathrm{ng} /$ mL: $68.8 \% ; \mathrm{p}<0.001$ ) was observed for both variants. Prolonged incubation resulted in decreased mRNA and protein expression $(\mathrm{p}=0.01)$ in CC/CTs only. Calcein-AM efflux remained higher in TTs $(29.90 \%$ vs. $41.26 \%$; $\mathrm{p}=0.016)$. Both relative CTGF mRNA and protein expression increased with Tac concentration (50 ng/ $\mathrm{mL}: 0.73 ; 300 \mathrm{ng} / \mathrm{mL} 0.79 ; \mathrm{p}=0.02$ ). Prolonged exposure nor CYP3A5 genotype had a significant effect on mean relative CTGF expression. However, subgroup analysis demonstrated lower CTGF expression after $72 \mathrm{~h}$ in $* 1$ carriers vs. non-carriers ( 0.58 vs. $0.81 ; \mathrm{p}=0.03)$. CC/CTs demonstrated a significant decrease after $72 \mathrm{~h}$, while CTGF expression in the TTs was significantly higher than CC/CTs.

Conclusions: Tac exposure in human PTC has no direct effect on the regulation of gene expression but results in decreasing functions of CYP3A5 and P-gp. Tac exposure in human PTC results in a concentration-dependent increase in CTGF expression (pro-fibrotic cytokine). Prolonged Tac exposure results in increased CTGF expression in PTC with lower CYP3A5 functional expression and higher P-gp function which might correspond with an increased risk for renal fibrosis in patients.

\section{8 \\ Role of AMP-Activated Protein Kinase in Renal Lipid Accumulation in Obesity-Induced Chronic Kidney Disease. Benefits of an Endurance Exercise Training in Mice}

Florian Juszczak ${ }^{1,2}$, Maud Valssembrouck', Thomas Zwakhals', Vincianne Jenart ${ }^{3}$, Olivia Botton ${ }^{2}$, Jean-François Gaussin ${ }^{2}$, Inès Jadot ${ }^{2}$, Alexandra Tassin ${ }^{3}$, Alexandre Legrand ${ }^{3}$, Nathalie Caron ${ }^{2}$, Anne-Emilie Declèves ${ }^{1}$

${ }^{1}$ Laboratory of Molecular Biology, University of Mons, Mons, Belgium; ${ }^{2}$ Laboratory of General Physiology, University of Namur, Namur, Belgium; ${ }^{3}$ Laboratory of Physiology and Respiratory Rehabilitation, University of Mons, Mons, Belgium

Background: The increased incidence of obesity in Western countries is largely due to a combination of sedentary lifestyle, associated with a high caloric intake and a lack of exercise. Furthermore, systematic reviews and meta-analysis demonstrated that obesity serves as an independent predictor of new-onset chronic kidney disease (CKD). Excessive caloric intake is related to lipid accumulation in adipose tissues but also ectopic lipid depositions in non-adipose tissues, leading to deleterious cellular responses. Our previous work demonstrated that in mice such ectopic depositions occur in the kidney following a high-fat diet (HFD), particularly in proximal tubular cells (PTC), leading to tubular cell structure impairments, inflammation and fibrosis. This was cor- 
related to a reduced activity of AMP-activated Protein Kinase (AMPK), a ubiquitous heterotrimeric enzyme that is considered a major energy sensor and a master regulator in cells.

Aim: This work determined for the first time the potential beneficial effect of endurance exercise training (EET) in long-term obese mice on renal lipotoxycity. Particularly, AMPK activity and lipid metabolism in isolated PTC was investigated.

Methods: A mouse model presenting long term HFD-induced CKD has been used. The impact of EET has been tested after 12 weeks on diet in already obese mice using a treadmill. During the protocol, mice were placed into metabolic cages for $24 \mathrm{~h}$-urine collection. Metabolic profiles regarding glucose and lipid metabolism were determined in urine and plasma samples. In addition, renal function was evaluated. Western blot, ELISA and RT-qPCR were used in kidney tissue and isolated PTC to delineate the AMPK pathway and key markers in lipid metabolism. This includes fatty acid synthase (FAS) and acetyl-CoA carboxylase (ACC) to investigate the lipogenesis pathway and canityl palmitoyltransferase 1 (CPT-1) to investigate the $\beta$-oxidation pathway.

Results: Mice fed a HFD exhibited a significant increase in body weight combined to increases in plasma levels of NEFA, triglycerides and cholesterol. Moreover, they also developed insulin resistance. ETT showed body weight stabilization and beneficial effects on plasma lipid profile in HFD mice. In addition, hyperglycaemia and insulin resistance were also clearly improved by exercise. Regarding the renal function, measurement of albuminuria and histological analysis suggested a more disparate result. Indeed, two populations were observed. A proportion of HFD mice submitted to EET showed positive effect on renal function along with an increase of AMPK activity while the second did not. Similar data were observed regarding ACC and FAS expression.

Conclusions: These results suggest that EET leads to beneficial effects to obesity-related metabolic disorders and to some extend in kidney in mice fed a HFD. This ongoing study gives us encouraging sets of data that will be further explored in order to better characterize the impact of EET on HFD-induced CKD.

\section{9}

\section{Association or Causation? Targeting Interstitial Fibrosis Attenuates the Loss of Renal Function in Chronic Kidney Disease}

\begin{abstract}
Stefanie Steiger ${ }^{1}$, Julia Felicitas Grill', Qiuyue Ma', Tobias Bäuerle" , Jutta Jordan², Michaela Smolle", Claudia Böhland ${ }^{4}$, Maciej Lech ${ }^{1}$, Hans-Joachim Anders ${ }^{1}$

${ }^{1}$ Klinikum der Universität München, Medizinische Klinik und Poliklinik IV, Division of Nephrology, Munich, Germany; ${ }^{2}$ Preclinical Imaging Platform, Institute of Radiology, Universitätsklinikum Erlangen, Erlangen, Germany; ${ }^{3}$ LudwigMaximilians Universität München, Biomedizinisches Centrum, Munich, Germany; ${ }^{4}$ Ludwig-Maximilians Universität München, Department of Radiation Oncology, Munich, Germany
\end{abstract}

That fibrosis may promote CKD has become a popular concept, although the few studies that tested for a causal relationship are inconsistent in terms of the renoprotective effect of antifi- brotic interventions. Here, we tested for causation by suppressing interstitial fibrosis through neutralizing TGF $\beta$ in mice with progressive nephrocalcinosis-related $\mathrm{CKD}$ and assessed the decline of glomerular filtration rate (GFR) as a primary endpoint by repetitively measuring GFR in each animal. Preemptive TGF $\beta$ inhibition substantially reduced interstitial fibrosis as indicated by magnetic resonance imaging, silver and $\alpha$-SMA staining, RT-qP$\mathrm{CR}$, and flow cytometry for pro-fibrotic macrophages. Suppressing interstitial fibrosis slowed the decline of GFR compared to treatment with control IgG1 when calculated using a one-compartment kinetic model (slope of $\mathrm{m}=-15.5$ vs. $\mathrm{m}=-18.9 \mu \mathrm{l} /$ $\mathrm{min} / 100 \mathrm{~g} \mathrm{BW} /$ day, $\Delta=18.0 \%$ ). This effect increased GFR at the end of the study ( $90.3 \mathrm{vs} .37 .4 \mu \mathrm{l} / \mathrm{min} / 100 \mathrm{~g} \mathrm{BW}, \Delta=18.9 \%$ ), and prolonged end stage renal disease (ESRD)-free renal survival by 3 days $(\Delta=17.6 \%)$. Employing a three-compartment model even increased effect size of GFR slope to $41.6 \%$, of GFR on day 14 to $37.9 \%$, and of ERSD-free survival to 9 days $(\Delta=39.1 \%)$. However, delayed onset of TGF $\beta$ inhibition from day 7 did not affect any of these parameters. We conclude, interstitial fibrosis contributes to CKD progression but the window-of-opportunity for antifibrotic drugs may be limited to preemptive therapy or to early stages of CKD.

\section{0}

\section{Acute Effects of a Single Intravenous Iron Dose on Endothelial Function and Oxidative Stress in Non-Dialysis Chronic Kidney Disease Patients. Protocol for a Cross-Over, Single-Center Study}

\author{
A.-M. Mehedinti ${ }^{1,3}$, L. Iosif ${ }^{3}$, I. Stoian ${ }^{2}$, S. Stancu ${ }^{1,3}$, G. Mircescu ${ }^{1,3}$, \\ C. Capusa $^{1,3}$ \\ ${ }^{1}$ Nephrology Department, "Carol Davila" University of \\ Medicine and Pharmacy, Bucharest, Romania; ${ }^{2}$ Biochemistry \\ Dept., "Carol Davila" University of Medicine and Pharmacy,

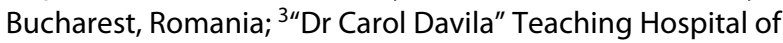 \\ Nephrology, Bucharest, Romania
}

Background. Since treatment guidelines for renal anemia often advocate intravenous (IV) iron, but experimental studies suggested that iron-induced free radicals production mediates endothelial cells damage, we addressed in clinical research the early changes in endothelial function after a single IV iron infusion, in the setting of chronic kidney disease (CKD). Previously, we found iron-related impaired arterial reactivity (assessed by flow-mediated dilatation of brachial artery - FMD) closely after an usual dose (200 mg) of IV iron sucrose, in a crossover study on sixty-three stages G3 to G5 non-dialysis CKD anaemic, iron-naïve subjects, as compared to a "blank" infusion with similar osmolarity (10\% glucose solution): median $\triangle \mathrm{FMD}-3.0(95 \% \mathrm{CI}-6.0$ to -2.2$)$ after IV iron vs. -0.8 ( $95 \% \mathrm{CI}-3.0$ to 1.0$), p=0.01$. Furthermore, in another crossover design which enrolled 31 analogous CKD patients and compared the acute effects of two iron formulations $(200 \mathrm{mg}$ IV iron sucrose and $500 \mathrm{mg}$ IV ferric carboxymaltose - FCM) with $250 \mathrm{~mL} 10 \%$ glucose, endothelial dysfunction seemed to occur only after iron sucrose [-2.3 (95\% CI -5.7 to 0.4$), p=0.01$ ], but not FCM [ $-0.8(95 \% \mathrm{CI}-3.5$ to 1.5$), p=0.27]$ versus the blank infusion [1.0 (95\% CI -2.0 to 2.5$)$ ]. Based on these rationale, we planned to in-
Abstracts 


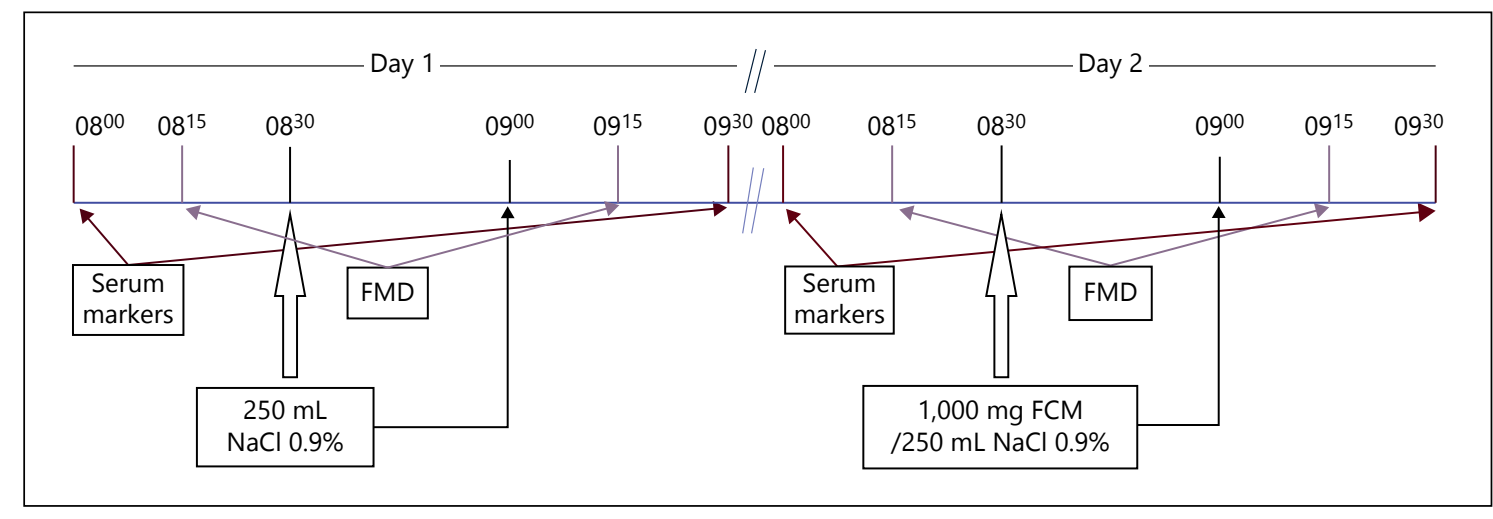

Fig. 1. (for Abstract 20).

vestigate the acute impact of a high dose of IV FCM on endothelial function, in relation to changes in oxidative and nitrosative status, also in non-dialysis CKD.

Methods. This prospective crossover single-center study is aimed to compare the effects of an infusion with $1000 \mathrm{mg}$ FCM in $250 \mathrm{~mL} 0.9 \%$ saline solution to those of a control infusion $(250 \mathrm{~mL}$ $0.9 \%$ saline solution), both administered over 30 minutes, 24 hours apart, the comparator first. Adults with CKD stages G3-G5, anemia and iron deficiency (all defined according to KDIGO criteria) comprise the studied population. Exclusion criteria are: active smoking, other causes of anemia, previous iron and epoetin therapy, contraindications for IV iron, active immunologic/inflammatory conditions, liver diseases or malignancies, pregnancy and breast feeding, severe anemia (hemoglobin $<7 \mathrm{~g} / \mathrm{dL}$ ), baseline FMD $<7 \%$, antioxidant supplements. Endothelium-dependent FMD (ultrasound) will be measured 15 minutes before and after each infusion as clinical parameter of endothelial function. In addition, 30 minutes before and after each infusion, serum markers of oxidative stress (both for reactive species generation and antioxidant system), nitric oxide activity, soluble ICAM-1 and VCAM1 (as signs of endothelial cells activation) will be assessed by spectrophotometry and ELISA (Figure 1).

The post-/pre-infusion differences $(\Delta)$ for each variable will be the main outcome of interest, while the Wilcoxon paired test will be the main method of comparison.

Discussion: To the best of our knowledge, the study assesses for the first time, concurrently in the same CKD subjects, the relationships among IV iron, oxidative stress, nitric oxide and endothelial dysfunction, so it is expected to provide new data on a controversial issue.

Ethical Approval: No. 139/2017 (Ethics Committee of the UMF “Carol Davila” Bucharest).

Funding: Partially supported by a research grant from SC Sanador SRL (contract 10488/2016).

Trial Registration: ClinicalTrials.gov Identifier NCT03388385.
21

\section{Sphingosine 1-Phosphate Receptor 5 (S1P5) in Renal Diseases}

\section{Timon Eckes, Josef Pfeilschifter, Alexander Koch}

Department of General Pharmacology and Toxicology, Goethe University Hospital Frankfurt, Frankfurt am Main, Germany

The bioactive lipid mediator sphingosine 1-phosphate (S1P) is generated on the intracellular side by phosphorylation of sphingosine which is catalyzed by two sphingosine kinases (SPHK), SPHK1 and SPHK2. With the help of transporters, S1P can cross the plasma membrane to act on five specific, $G$ protein-coupled receptors, $\mathrm{S} 1 \mathrm{P} 1-5 . \mathrm{S} 1 \mathrm{P}$ receptor activation is known to induce the expression of profibrotic mediators such as connective tissue growth factor (CTGF) and thus might play a role in the progression of renal fibrosis. From the five identified S1P receptors, the S1P5 receptor is the least characterized and the biological functions are poorly understood. Previously, we showed that S1P5 activation contributes to the transforming growth factor beta (TGF- $\beta 2$ ) induced CTGF expression in human mesangial cells. Here, we investigated the role of S1P5 in an in vivo model of tubulointerstitial fibrosis. Our results clearly demonstrate that S1P5 knockout mice had lower plasma creatinine levels compared to wildtype mice in adenine induced renal fibrosis. Further, the renal expression of several fibrotic markers was diminished in S1P5 knockout mice compared to wildtype mice. Taken together, S1P5 seems to play an essential role in the progression of renal fibrosis and might be a promising target for future treatment. 


\section{2}

\section{Serotonin Receptor Expression in the Normal Adult Human Kidney and in Glomerular Diseases}

\author{
Claudia R.C. van Roeyen ${ }^{1}$, Jenny Vu' ${ }^{1}$, Bart Smeets ${ }^{2}$, \\ Kerstin Saalmüller ${ }^{1}$, Marcus J. Moeller ${ }^{1}$, Maja T. Lindenmeyer ${ }^{3}$, \\ Clemens D. Cohen ${ }^{3}$, Luc Maroteaux ${ }^{4}$, Hermann Josef Gröne ${ }^{5}$, \\ Jürgen Floege ${ }^{1}$, Tammo Ostendorf', Gerald Braun ${ }^{1}$ \\ ${ }^{1}$ Division of Nephrology, RWTH Aachen University, Aachen, \\ Germany; ${ }^{2}$ Division of Pathology, Radboud University, \\ Nijmegen, Netherlands; ${ }^{3}$ Division of Nephrology, Medical \\ Clinic and Policlinic IV, University of Munich, Munich, Germany; \\ ${ }^{4}$ Institut du Fer a Moulin UMR-S839 INSERM/UPMC, Paris, \\ France; ${ }^{5}$ Department of Cellular and Molecular Pathology, \\ German Cancer Research Center, Heidelberg, Germany
}

Serotonin (5-HT) is the phylogenetically oldest neurotransmitter and acts via specific serotonin receptors. In mammalians 14 different serotonin receptors exist. The serotonin receptors 5-HT2a and -2b are G-protein-coupled and mediate vasoconstriction and hypertension. 5-HT2a is also engaged in blood coagulation and wound healing. Little is known about the function of 5-HT and its receptors 5-HT2a and -2b in the normal kidney or during renal disease. Localization of 5-HT2a and -2b was analysed by immunohistochemistry in the human healthy kidney and during renal disease. Additionally, in vitro studies were performed to identify the role of $5-\mathrm{HT} 2 \mathrm{a}$ and $-2 \mathrm{~b}$ in primary murine parietal epithelial cells (PECs). In the normal human kidney (living donor biopsies), 5-HT2a and -2b localized to a subgroup of tubular and glomerular parietal epithelial cells. In all renal diseases investigated, 5-HT2a and -2b tubular expression was maintained. In glomeruli, the number of 5-HT2b positive cells increased with disease. By immunofluorescence glomerular 5-HT2b positive cells could be identified as PECs. In biopsies of patients with IgA nephropathy and rapidly progressive glomerulonephritis (RPGN) patients, the cell diameter of 5-HT2b-positive PECs was significantly increased in comparison to receptor-negative PECs. In primary PECs in vitro, activation of 5-HT2a and -2b induced overexpression of PEC activation markers VCAM and CXCR-4. In addition, 5-HT2b but not -2a increased Col4a1 mRNA expression. In summary, we demonstrate the constitutive expression of 5-HT2a and 5-HT2b in adult human kidney with glomerular upregulation in different renal diseases. Our studies identify PECs as the primarily cell type involved in serotonin receptor upregulation in glomerular disease. Further studies will focus on the potential pathophysiological role of these receptors.

\section{3 \\ The Loss of Podocytes in Glomerular Hypertension Depends on a Preceding Failure of the Mesangium \\ Wilhelm Kriz}

Department of Neuroanatomy, Medical Faculty Mannheim, University of Heidelberg, Mannheim, Germany

Glomerular hypertension damages the glomerulus leading to progressive glomerular disease. It is widely believed that increased perfusion pressure exposes the tuft to increased tensile (expansile) stress challenging the adhesion of podocytes to the GBM favo uring their detachment. This interpretation is based on the view that podocyte foot processes, like pericyte processes, actively counteract the pressure-driven expansion of the GBM and fail in hypertension. Recent findings have compromised this view. Podocyte foot processes need their actin-based cytoskeleton to maintain the complex pattern of interdigitation and to adapt to pressure derived area changes of the GBM but are unable to counteract pressure driven expansion. Instead, counteraction of tensile stress to the filtration barrier seems to be almost exclusively performed by the GBM. The GBM displays a non-linear elasticity (strain stiffening) [1] meaning that its distensibility progressively decreases with increasing distension and finally reaches a limit. Thus, the expansion of a glomerular capillary and thus the expansile challenge to podocytes has an upper limit $[2,3]$. Podocytes are protected from any unphysiological pressure rises by the GBM. As described in many previous studies, hypertension leads to focal damage in the mesangium. The folding pattern of the GBM is maintained by mesangial cells that insert to the GBM; their contractile tonus permanently counteracts the expansile forces of perfusion pressure. Disruptions of mesangial cell-GBM-connections by excessive tensile stress from hypertension lead to a bulging of capillaries and mesangial spaces, together to local expansions of the tuft. The danger that results from such local derangements of tuft architecture to podocytes has never been adequately recognized. Here it is shown that damage of mesangial cell-GBM-connections are consistently followed by displacements of capillary loops and corresponding podocytes. Most spectacular are the cases in which capillary loops become dislocated towards the urinary orifice. The corresponding podocytes are dragged by shear stress toward and into the urinary orifice and are either finally lost by detachment and spoiled away with the urine or lead to the formation of a tip lesion. Even if less obvious also the development of vascular pole associated FSGS seems to crucially depend on a preceding mesangial damage. Also in these cases a bulging of expanded capillary loops is regularly seen that leads to contacts of the corresponding podocytes with the parietal epithelium. Such contacts lead, as described previously [4], to the formation of tuft adhesions, the committed lesion to start the pathway to FSGS. In conclusion, the development of FSGS in consequence of glomerular hypertension emerges from a combined failure of the mesangial cells and podocytes, whereby the mesangial damage is due to increased tensile stress, the podocyte damage due to displacement and shear stress. The same dependence of podocyte damage on a preceding mesangial damage is also seen in any glomerular diseases (e.g. nephritis), in which the mesangial cell-GBM-connections are directly damaged independent of hypertension. 


\section{References}

1 Janmey PA, Miller RT: Mechanisms of mechanical signaling in development and disease. J Cell Sci 2011;124(Pt 1):9-18.

2 Kriz W, Lemley KV: Potential relevance of shear stress for slit diaphragm and podocyte function. Kidney Int 2017;91(6):1283-1286.

3 Kriz W, Lemley KV: Mechanical challenges to the glomerular filtration barrier: adaptations and pathway to sclerosis. Pediatr Nephrol 2017; 32(3):405-417.

4 Le Hir M, Keller C, Eschmann V, Hähnel B, Hosser H, Kriz W: Podocyte bridges between the tuft and Bowman's capsule: an early event in experimental crescentic glomerulonephritis. J Am Soc Nephrol 2001;12(10): 2060-2071.

\section{4}

\section{Single-Cell Transcriptome Profiling of Kidney Glomeruli in Normal Human and Mouse}

Bing He, Ping Chen, Jing Guo, Sonia Zambrano Sevilla,

Integrated Cardio Metabolic Centre (ICMC), Department of

Medicine Huddinge at Novum, Karolinska Institute, Huddinge,

Sweden

Glomerular disorders account for $>80 \%$ of end-stage renal disease cases. However, the pathogenesis of these diseases is poorly understood. In this study, we carried out single cell RNA-seq of glomeruli from human donor biopsies and wild-type mouse kidneys. Our aim was to establish a reproducible method for single cell preparation and construct a single cell transcriptome atlas of normal glomeruli.

To prepare single cells, we first isolated glomeruli from whole kidney. For human kidney biopsy, we performed a two-sieving process. For mouse kidney, magnetic bead perfusion was needed. Enriched glomeruli were then enzymatically digested for single cell dissociation. Viable single cells were unbiasedly distributed into 384well plates by the FACS sorter. Fulllength cDNA libraries were generated using the Smart-seq 2 protocol followed by sequencing and data analysis.

The protocol for preparing viable single cells was validated by analyzing the global RNA integrity. As far, we have sequenced about 700 cells passing quality control. In global transcriptome analysis, 378 cells of adult mouse glomeruli were categorized to 7 distinct clusters. Using well-known cell type specific markers, all three glomerular structural cell types, endothelial cells (56\%), podocytes (10\%) and mesangial cells (5\%), were included. Interestingly, almost $15 \%$ of immune cells (macrophages, $\mathrm{T}$ cells and $\mathrm{B}$ cells) and $4 \%$ of proximal tubular cells were identified. More than 300 human cells displayed a similar cluster pattern to mouse, but the difference was a very low number of podocytes and mesangial cells. Based on these data, we identified a number of cell-type specific known and novel genes in diverse cell types. In the endothelial group, several genes highly expressed in human were undetectable in mouse, and this population showed a notably heterogeneous distribution. Further, we observed a unique expression pattern in which genes specific for pericytes or smooth muscle cells were simultaneously expressed in mouse mesangial cells.

Our preliminary results provide insight into glomerular function at the single cell resolution. This study may represent a new avenue for better understanding pathogenesis of glomerular diseases.

\section{5}

Gprc5a - A Novel Podocyte Target Plays a Pathogenic Role in the Development of Diabetic Nephropathy

Sonia Zambrano Sevilla, Xiaojie Ma, Angelina Schwarz, Jaakko Patrakka

$\mathrm{KI} / \mathrm{AZ}$ Integrated Cardio Metabolic Centre (ICMC), Department of Laboratory Medicine, Karolinska Institutet, Stockholm, Sweden

G-protein coupled receptors (GPCRs) have been in the past targeted successfully by pharmaceutical industry and could be attractive targets also in glomerular disease processes. In this study, we aimed to identify glomerulus-associated GPCRs that could be novel targets to treat diabetic nephropathy (DN). Large-scale molecular profiling of kidney tissue revealed an orphan GPCR, Gprc5a, to be one of the most highly expressed GPCRs in the human glomerulus. Gprc5a was highly specific to podocyte cells and its expression was significantly depleted in $\mathrm{DN}$. We generated a Gprc5a knockout mouse line (KO) and observed that glomerular filtration barrier and podocytes were intact in young Gprc5a-deficient animals. However, in aging animals we detected thickening of the glomerular basement membrane and activation of mesangial cells, two hallmark signs of human DN. Moreover, we observed activation of EGFR and TGFB signaling in KO glomeruli. In streptozocin-induced diabetes model, KO mice showed more albuminuria, podocyte foot process effacement and glomerular fibrosis than controls. In cell culture, ectopic overexpression of Gprc5a in podocytes inhibited EGF induced EGFR activation and Smad2/3 phosphorylation. Consistently, TGFB1 and its downstream extracellular matrix expression were also blunted. In line with this, knocking down Gprc5a in cell culture using siRNA had opposite effects. We conclude that Gprc5a and its downstream signaling pathways play a protective role in maintaining podocyte and glomerular health. We propose that manipulation of podocyte signaling via Gprc5a may represent a novel, targeted therapeutic option for DN.

\section{6}

\section{Podocyte-Expressed STAT5 Confers Protection During Experimental Glomerulonephritis and Adriamycin Nephropathy in Mice}

\section{K. Louis, A. Niasse, A. Corchia, S. Placier, Y.C. Xu-Dubois, S. Vandermeersch, E. Rondeau, L. Mesnard, Y. Luque}

UMR_S 1155, Unité mixte Inserm-Université 'Des maladies rénales rares aux maladies fréquentes, remodelage et réparation' Hôpital Tenon - Bâtiment Recherche, Paris, France

Glomerular diseases are a leading cause of chronic kidney failure and the podocyte is one of the main target of these diseases. We previously evidenced a protective role for a podocyteexpressed immune receptor such as the common gamma chain $(\gamma \mathrm{C})$ 


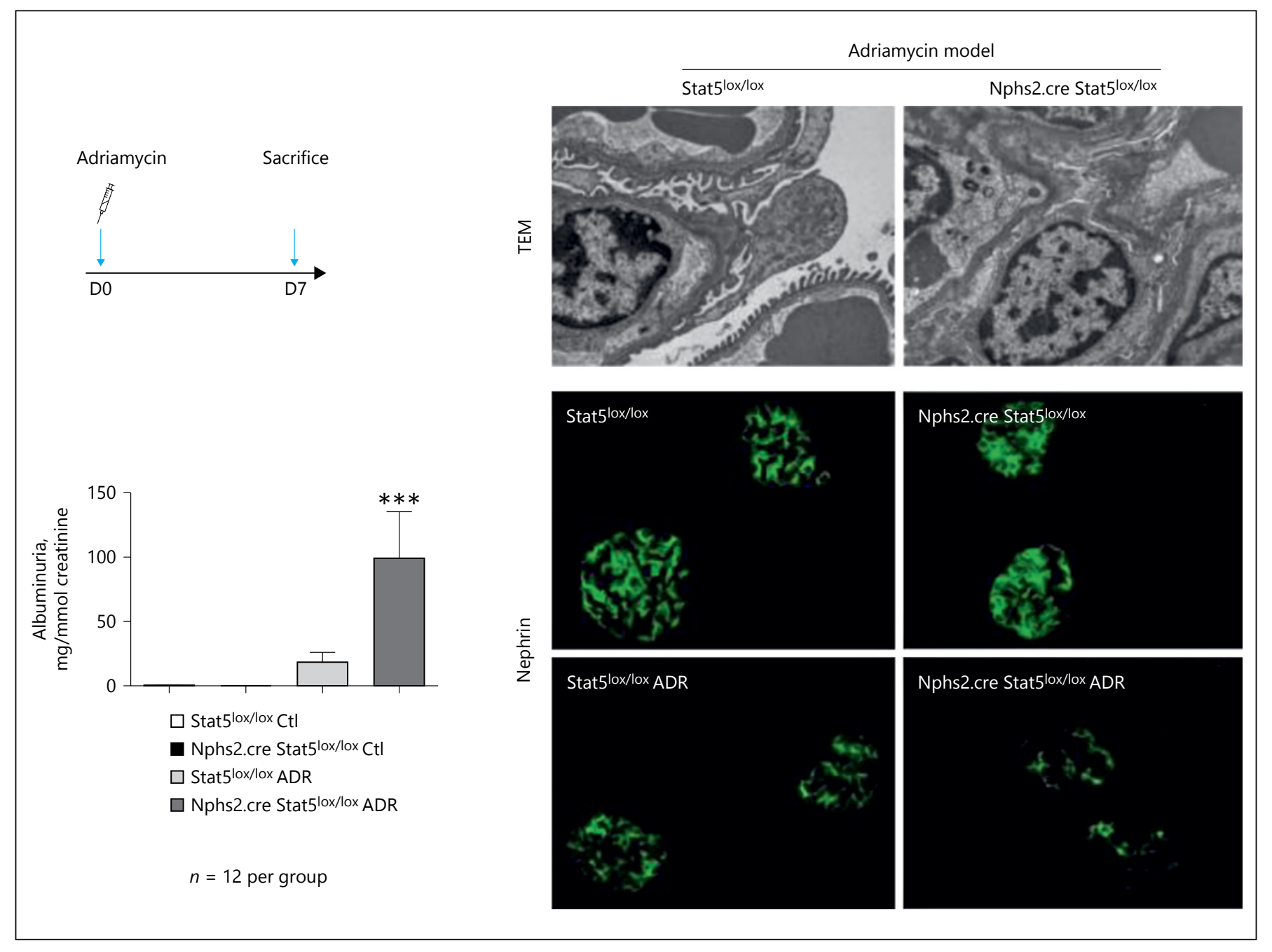

Fig. 1. (for Abstract 26).

during glomerulonephritis [1]. We also found that STAT5, a transcriptional factor classically described and activated downstream $\gamma \mathrm{C}$ in T cells is upregulated in podocytes during glomerulonephritis. Hitherto, STAT5 role in podocyte remains unclear. Using mice with a podocyte-specific deletion of Stat5, we analyzed the role of STAT5 in two experimental models of glomerular diseases. First, during crescentic glomerulonephritis, podocytic-STAT5 deficient mice developed increased proteinuria compared to their wild-type littermates. Second, during adriamycin induced-nephropathy, the absence of podocyte STAT5 leads to increased albuminuria and severe podocytic injuries in comparison to controls (Figure 1). Moreover, podocytic lesions were associated with loss of podocyte differentiation markers such as WT1 especially in podocytic-STAT5 deficient animals. Renal T-cells and macrophages infiltration were not affected by the deletion of podocytic Stat5.

Taking together, our results suggest a yet unsuspected protective role of podocytic $\gamma \mathrm{C}$ /STAT5 signaling during glomerular diseases.

\section{Reference}

1 Luque Y, Cathelin D, Vandermeersch S, Xu X, Sohier J, Placier S, et al: Glomerular common gamma chain confers B- and T-cell-independent protection against glomerulonephritis. Kidney Int 2017;91(5):11461158. 


\section{7}

\section{Targeting CD146 Protects Against Experimental Glomerulonephritis in Mice}

Ahmed Abed ${ }^{1,2}$, Aurélie S. Leroyer ${ }^{3}$, Panagiotis Kavvadas ${ }^{1}$, Florence Authier ${ }^{1}$, Richard Bachelier ${ }^{3}$,

Alexandrine Foucault-Bertaud ${ }^{3}$, Nathalie Bardin ${ }^{3}$,

Noémie Jourde-Chiche ${ }^{3,4}$, Stéphane Burtey ${ }^{3,4}$,

Marcel Blot-Chabaud ${ }^{3}$, Françoise Dignat-George ${ }^{3}$, Christos E. Chadjichristos ${ }^{1,2}$

'INSERM UMR-S1155, Batiment Recherche, Tenon Hospital, 4 rue de la Chine 75020 Paris, France; ${ }^{2}$ Sorbonne Universite's, UPMC Univ Paris 6, Paris, France; ${ }^{3}$ Aix-Marseille Univ, INSERM UMR-S 1076, UFR Pharmacy, Marseille, France; ${ }^{4}$ Aix-Marseille Univ, Department of Nephrology, AP-HM Hôpital de la

Conception, Marseille, France

Glomerulonephritis (GN) includes a variety of renal pathologies which may progress to endstage renal disease. CD146 is an endothelial junctional adhesive molecule which expression is increased in human glomerular diseases. However, the pathological significance of this overexpression remains unknown. Thus, the aim of our study was to investigate the role of CD146 in the nephrotoxic serum-induced GN (NTS-GN) model in mice.

During the progression of NTS-GN, CD146 expression was highly induced within injured kidneys. Interestingly, CD146 knock-out mice showed improved renal function as both proteinuria and blood urea nitrogen levels were highly reduced, two weeks after the induction of the disease. Furthermore, at the same time point, histological lesions such as crescent formation, tubular dilation, monocyte and lymphocyte infiltration and interstitial renal fibrosis were strongly reduced compared to wild type animals.

Colocalization experiments with markers for different glomerular cells showed that CD146 expression was mainly increased within the injured endothelium of the glomerular tuft. Consequently, we generated a new conditional transgenic strain in which CD146 was specifically deleted in the vascular endothelium upon tamoxifen administration. These mice showed substantial preservation of renal structure and function compared to WT animals.

Taken together, our data showed that endothelial CD146 plays a major role in the progression of GN and may represent a novel therapeutic target to reduce glomerular damage and renal fibrosis.

\section{8}

Palladin Plays an Important Role for Podocyte Foot Process Morphology and Function in Mice

Nadine Artelt ${ }^{1}$, Alina M. Ritter ${ }^{1}$, Panagiotis Kavvadas ${ }^{2}$, Rabea Schlüter ${ }^{3}$, Carol Otey ${ }^{4}$, Marie Louise Bang ${ }^{5,6}$, Jens van den Brandt ${ }^{7}$, Christos E. Chadjichristos ${ }^{2}$, Christos Chatziantoniou ${ }^{2}$, Karlhans Endlich ${ }^{1}$, Nicole Endlich ${ }^{1}$

${ }^{1}$ Department of Anatomy and Cell Biology, University Medicine Greifswald, Greifswald, Germany; ${ }^{2}$ National Institute for Health and Medical Research Unité Mixte de Recherche-S1155, Tenon Hospital, Paris, France; ${ }^{3}$ Imaging Center of the Department of Biology, University of Greifswald, Greifswald, Germany; ${ }^{4}$ UNC Department of Cell Biology and Physiology, Chapel Hill, North Carolina, USA; ${ }^{5}$ Institute of Genetic and Biomedical Research, UOS Milan, National Research Council, Milan, Italy; ${ }^{6}$ Humanitas Clinical and Research Center, Rozzano, Milan, Italy; ${ }^{7}$ ZSFV; University Medicine Greifswald, Greifswald, Germany

Effacement of interdigitating podocyte foot processes is the major cause for a leaky filtration barrier in the glomerulus resulting in proteinuria followed by the development of chronic kidney diseases. Since the complex podocyte foot process morphology is highly dependent on the intact actin cytoskeleton, we already studied the role of the actin-bundling and focal adhesion protein palladin [1].

Since a knockout of palladin is lethal at a very early point in time during development, we generated a podocyte-specific palladin knockout mouse (PodoPalld-KO) to examine the role of palladin in podocytes in vivo. These mice developed glomeruli with a disturbed morphology, podocytes with sub-podocytes spaces as well as a mild effacement accompanied by a reduced nephrin expression, but no significant proteinuria was observed. However, after NTS injection PodoPalld-KO mice showed aggravated proteinuria as well as a significant impaired podocyte morphology compared to the NTS treated control mice.

As it is known that the genetic background of mice significantly influences the manifestation of kidney diseases, we backcrossed the PodoPalld-BL6-KO mice to the 129S1 background which is reported to be more susceptible for the development of kidney diseases. We found that the podocyte morphology and the expression of nephrin as well as of Pdlim2 and LASP-1, two palladin-binding proteins, were much more affected in PodoPalld-129S1-KO mice in contrast to PodoPalld-BL6-KO mice.

Taken together, our results demonstrate the important role of palladin in podocyte architecture and renal function.

Reference

1 Endlich N, Schordan E, Cohen CD, Kretzler M, Lewko B, Welsch T, Kriz W, Otey CA, Endlich K; European Renal cDNA Bank Consortium: Palladin is a dynamic actin-associated protein in podocytes. Kidney Int 2009;75(2):214-226. 


\section{9}

\section{Studying the Role of Fibronectin in Mechanically Stressed Podocytes}

Felix Kliewe ${ }^{1}$, Soeren Kaling ${ }^{1}$, Henriette Loetzsch ${ }^{1}$, Sindy Schroeder ${ }^{1}$, Christian Scharf ${ }^{2}$, Kerstin Amann ${ }^{3}$, Maja T. Lindenmeyer ${ }^{4}$, Clemens D. Cohen ${ }^{4}$, Karlhans Endlich ${ }^{1}$, Nicole Endlich ${ }^{1}$

${ }^{1}$ Department of Anatomy and Cell Biology, University Medicine Greifswald, Greifswald, Germany; ${ }^{2}$ Department of Otorhinolaryngology (ENT); University Medicine Greifswald, Greifswald, Germany; ${ }^{3}$ Department of Nephropathology; University Medicine Erlangen, Erlangen, Germany; ${ }^{4}$ Nephrological Center, Medical Clinic and Policlinic IV, University of Munich, Munich, Germany

Diabetic nephropathy (DN) is one of the leading diseases in Western societies often resulting in a complete loss of kidney function called end stage renal disease (ESRD). DN is characterized by proteinuria, glomerulosclerosis and foot process effacement of podocytes. Moreover, $\mathrm{DN}$ is associated with a loss of viable podocytes probably caused by glomerular hypertension. Since podocytes are postmitotic and unable to proliferate, the loss of podocytes cannot be compensated.

Podocytes that are attached to the outer aspect of the glomerular capillaries by their interdigitating foot processes are essential for an intact glomerular filtration barrier. We have shown that podocytes are mechanosensitive and reorganize their actin cytoskeleton in response to mechanical stress in vitro [1]. Furthermore, we have found that mechanical stress could induce detachment of podocytes in vitro [2].

In this study, we demonstrate that the extracellular matrix protein fibronectin is essential for the attachment of podocytes during mechanical stress. By qRT-PCR as well as by LC-MS (Liquid Chromatography-Mass Spectrometry) we found a significant upregulation of fibronectin due to mechanical stress (3 days, $0.5 \mathrm{~Hz}$ and $5 \%$ extension). To study the role of fibronectin in cultured podocytes under mechanical stress, fibronectin was knocked down (FN-KD) by specific siRNA. Additionally, we established a fibronectin knockout podocyte cell line (FN-KO) by CRISPR/Cas9.

During mechanical stress, we observed a significant loss of FN$\mathrm{KD}$ as well as $\mathrm{FN}-\mathrm{KO}$ podocytes $(>80 \%)$ due to mechanical stress compared to the control cells. Furthermore, FN-KO podocytes showed a significant downregulation of the focal adhesion proteins talin, vinculin and paxillin and a reduced cell spreading indicating an important role of fibronectin for the adhesion of cultured podocytes. Analyzing kidney sections of patients suffering from DN and FSGS, we found a significant upregulation of fibronectin in contrast to control biopsies.

In summary, we show that fibronectin plays an important role in the adaptation of podocytes to mechanical stretch.

\section{References}

1 Endlich N, Kress KR, Reiser J, Uttenweiler D, Kriz W, Mundel P, Endlich $\mathrm{K}$ : Podocytes respond to mechanical stress in vitro. J Am Soc Nephrol 2001;12(3):413-422.

2 Schordan S, Schordan E, Endlich K, Endlich N: AlphaV-integrins mediate the mechanoprotective action of osteopontin in podocytes. Am J Physiol Renal Physiol 2011;300(1):F119-F132.

\section{0 \\ Adriamycin Does Not Induce Podocyte Damage Per Se in Zebrafish Larvae}

\author{
Maximilian Schindler, Antje Blumenthal, Karlhans Endlich, \\ Nicole Endlich
}

Department of Anatomy and Cell Biology, University Medicine Greifswald, Greifswald, Germany

The prevalence of glomerulopathies that lead to proteinuria and end-stage renal disease is increasing worldwide. Most of these glomerulopathies are associated with an impairment of podocytes followed by podocyte depletion and podocyte foot process effacement. Due to a limited understanding of the causes responsible for podocytopathies, curative therapies and drugs are still missing.

Zebrafish larvae develop a simple kidney (pronephros) composed of a pair of tubules connected to a single Glomerulus which is highly similar to glomeruli of mice and humans. This is why the zebrafish larva is well established as an easy and quick model to study kidney function in a living organism.

In order to induce podocyte damage in rodents as well as in zebrafish larvae, Adriamycin (ADR), a postulated nephrotoxic drug, was used in the past. Since ADR $(10-20 \mu \mathrm{M})$ generated very heterogeneous and not reliable phenotypes of the developing larvae when added to the tank water of fertilized zebrafish eggs, we studied the influence of ADR directly on zebrafish larvae at later time points to exclude developmental side effects.

For this purpose, we added ADR in different concentrations $(20 \mu \mathrm{M}, 40 \mu \mathrm{M}, 60 \mu \mathrm{M})$ to the tank water of ET zebrafish larvae at day 7 post fertilization (dpf) for 48 hours. This specific ET zebrafish strain is completely translucent and expresses eGFP under the $w t 1 a$ promotor specifically in podocytes [1].

Subsequently, histological sections followed by haematoxylin and eosin or methylene blue staining showed no differences of the glomerular morphology due to ADR treatment. Furthermore, the expression of the slit membrane-specific proteins nephrin and podocin as well as the eGFP fluorescence of podocytes did not change during ADR-treatment analyzed by immunhistology and qRT-PCR. Moreover, electron microscopic analysis of the glomerulus showed no differences between ADR-treated $(60 \mu \mathrm{M})$ and control larvae regarding the structure of the endothelium, the thickness of the GBM and the morphology of the podocyte foot processes. In accordance with these results, ADR-treated larvae barely developed edema, a hallmark of kidney failure indicating that $\mathrm{ADR}$ is not a podocyte damaging drug per se in zebrafish larvae.

In conclusion, the impairment of the glomerular function of ADR-treated zebrafish larvae observed in the past is most likely caused by development side effects and not by a direct effect on podocytes.

\section{Reference}

1 Endlich N, Simon O, Göpferich A, Wegner H, Moeller MJ, Rumpel E, Kotb AM, Endlich K: Two-photon microscopy reveals stationary podocytes in living zebrafish larvae. J Am Soc Nephrol 2014;25(4):681-686. 


\section{1}

\section{Screening Candidate Genes for Diabetic Kidney Disease in Danio Rerio}

\begin{abstract}
Darrell Andrews $^{1,2}$, Eoin Brennan ${ }^{1,2}$, Lynne Staggs ${ }^{3}$, Patricia Schroder ${ }^{3}$, Janina Mueller Deile ${ }^{4}$, Ross Doyle ${ }^{1,2}$, Peter Conlon ${ }^{5}$, Denise Sadlier ${ }^{6}$, Caitriona McEvoy ${ }^{1,2}$, The GENIE Consortium, Mario Schiffer ${ }^{3,4}$, Catherine Godson ${ }^{1,2}$

${ }^{1}$ Diabetes Complications Research Centre, Conway Institute of Biomolecular and Biomedical Research, University College Dublin, Ireland; ${ }^{2}$ UCD School of Medicine, University College Dublin, Ireland; ${ }^{3}$ Mount Desert Island Biological Laboratory, Maine, USA; ${ }^{4}$ Department of Nephrology, Hannover Medical School, Hannover, Germany; ${ }^{5}$ Department of Nephrology, Beaumont Hospital, Dublin, Ireland; ${ }^{6}$ Mater Misericordiae University Hospital, Dublin, Ireland
\end{abstract}

Diabetic kidney disease (DKD) is the leading cause of end stage renal disease (ESRD) and organ failure worldwide. Current therapies are inadequate at best slowing progression of disease but not arresting or reversing. Defining the genetic architecture of DKD is critical to unravelling the underlying mechanisms. Through the GENIE consortium, we are investigating genetic associations with DKD. We have identified several potential candidates but causation is difficult to infer without robust screening tools. The zebrafish, danio rerio, has emerged as a useful organism for the study of renal pathophysiology owing to its structural and genetic homology to the mammalian kidney. Here we describe a robust and versatile screen for investigating the function of candidate genes in danio rerio. Genetic candidates were identified by GWAS studies in a case $(n=2,861$ individuals with T1DM) vs. control $(n=12,418$ individuals with T1DM and no renal disease 15 years post-diagnosis) approach. Genetic candidates reaching genome wide significance $\left(\mathrm{p}<1 \times 10^{-8}\right)$ were included for functional follow up. In a study of one genetic candidate, $\mathrm{Tg}$ (l-fabp:DBP:EGFP) zebrafish embryos were injected with a morpholino against our candidate gene, control morpholino or CD2AP as a positive control. Tg(lfabp:DBP:EGFP) is a transgenic zebrafish strain that expresses vitamin $\mathrm{D}$ binding protein fused to enhanced green fluorescent protein (DBP:EGFP) into the circulation of the fish. The fusion protein has a molecular weight of $78 \mathrm{kDa}$, comparable to human albumin. Compromised glomerular barrier integrity leads to loss of the fusion protein from the circulation which can be quantified by fluorescence imaging of the retinal plexus. There was a significant loss of retinal fluorescence in the knockdown group $(n=60)$ vs control $(n=54)$, equivalent to that of our positive control CD2AP. The molecular function of this gene was investigated by examining its downstream targets as well as kidney specific markers in the knockdown model using gene expression and Western blotting analysis respectively. Finally, ultrastructural changes in the glomerular barrier was visualised using electron microscopy, revealing pronounced podocyte foot process effacement in knockdown fish. Screening of a cohort of renal cell lines confirmed high levels of gene expression in podocytes. This screening tool using Tg(l-fabp:DBP:EGFP) transgenic zebrafish is a versatile and comprehensive method to investigate alterations in glomerular function associated with DKD in humans and provides both functional and molecular information about probable genetic candidates.

\section{2}

Long Non-Coding RNAs in Diabetic Nephropathy

Simone Reichelt-Wurm ${ }^{1}$, Dominik Chittka ${ }^{1}$, Tobias Wirtz', Sebastian Beck' ${ }^{1}$, Aristidis Charonis' ${ }^{2}$,Markus Kretz ${ }^{3}$, Bernhard Banas ${ }^{1}$, Miriam C. Banas ${ }^{1}$

${ }^{1}$ Department of Nephrology, University Hospital Regensburg, Regensburg, Germany; ${ }^{2}$ Biomedical Research Foundation of the Academy of Athens, Athens, Greece; ${ }^{3}$ Institute of Biochemistry, University of Regensburg, Regensburg, Germany

Background and Purpose: Diabetic nephropathy (DN) is the leading cause for end-stage renal disease in both industrial and developing countries. Therefore, research on this subject is essential regarding the growing prevalence, the often devastating clinical course, and the extremely high costs for the global health systems. To analysze the underlying mechanisms we use the inherently insulin-resistant mouse strain BTBR with the ob/ob leptin-deficiency mutation. These mice develop severe type 2 diabetes with a renal phenotype which closely resembles human DN. By 22 weeks diffuse mesangial sclerosis, focal arteriolar hyalinosis, mesangiolysis, and mild interstitial fibrosis were present. In recent years, evidence emerged that in humans $98 \%$ of all transcripts have no protein coding potential. A significant portion of these transcripts are long non-coding (lnc) RNAs. Research revealed multiple regulatory functions of IncRNAs in development, cancer, and various diseases including pre- and posttranscriptional control, splicing, and genomic imprinting. As demonstrated before, lncRNAs are differentially expressed in the UUO mouse model of renal fibrosis [1]. Our aim ist to study the relevance of lncRNAs in development and progression of DN regarding in particular their role for the mesangium.

Methods: We isolated glomeruli from 4, 8, 16, and 24 weeks old BTBR WT and BTBR ob/ob mice and extracted the RNA. The Affymetrix GeneChip Mouse Whole Transcriptome Assay ${ }^{\circledR}$ was applied for differential expression analysis of 65.956 gene loci.

Results: Based on the microarray we ascertained 3253 coding and non-coding transcripts in total being differentially expressed in all 4 age groups. Comparing the mRNA expression profile of 24 weeks old BTBR ob/ob mice to a human data set of diabetic glomeruli we found a high degree of similarity. So for instance, around $70 \%$ of differentially expressed coding genes were downregulated with numerous matches in both data sets [2]. Regarding the lncRNAs' expression profiles (esp. in 24 weeks old mice) much more transcripts were upregulated in diabetic animals. One differentially expressed non-coding transcript in this age group is called Neat1 (Nuclear Paraspeckle Assembly Transcript 1) - a lncRNA that was shown to be an important factor for cell viability but also downregulated in hearts from diabetic Akita mice. However, the vast majority of lncRNAs has not been characterized so far. Therefore, differentially expressed lncRNAs were examined according to their genomic locus. We analyzed if there are genomic "hot spots" where many lncRNAs can be mapped to the same locus. Furthermore, we quantified the amount of lncRNAs being intergenic, neighboring a coding gene, or overlapping in sense or antisense direction with another gene locus. The data set of these coding genes allowed gene ontology analyses to establish a connection between (coding) gene expression and potentially cis-aciting lncRNAs. 
Future Aim: To identify and characterize lncRNAs potentially involved in the development and progression of $\mathrm{DN}$ we plan to conduct in vitro knockdown and overexpression experiments followed by gene expression analysis e.g. using microarray technology.

\section{References}

1 Arvaniti E, Moulos P, Vakrakou A, Chatziantoniou C, Chadjichristos C, Kavvadas P, Charonis A, Politis PK: Whole-transcriptome analysis of UUO mouse model of renal fibrosis reveals new molecular players in kidney diseases. Sci Rep 2016;6:26235.

2 Chittka D, Banas B, Lennartz L, Putz FJ, Eidenschink K, Beck S, Stempfl T, Moehle C, Reichelt-Wurm S, Banas MC: Long-term expression of glomerular genes in diabetic nephropathy. Nephrol Dial Transplant 2018, Doi: 10.1093/ndt/gfx359 [Epub ahead of print].

\section{3}

\section{Down-Regulation of Human LINC01187 IncRNA Gene Expression is Associated with Nephropathies}

\author{
Dora Manolakou ${ }^{1}$, Valeria Kaltezioti ${ }^{1}$, Elena Arvaniti ${ }^{1}$, \\ Hara Gakiopoulou ${ }^{2}$, Panagiotis Kavvadas ${ }^{3}$, \\ Christos Chatziantoniou ${ }^{3}$, Miriam Banas ${ }^{4}$, \\ Simone Reichert-Wurm ${ }^{4}$, Maja T. Lindenmeyer ${ }^{5}$, \\ Clemens D. Cohen ${ }^{5}$, Peter Boor ${ }^{6}$, Sonja Djudjaj ${ }^{6}$, Panos Politis ${ }^{1}$, \\ Aristidis Charonis $^{1}$ \\ ${ }^{1}$ Biomedical Research Foundation of the Academy of Athens, \\ Athens, Greece; ${ }^{2}$ Department of Pathological Anatomy, \\ University of Athens Medical School, Athens, Greece; ${ }^{3}$ INSERM \\ UMRS 1155, Tenon Hospital, Paris, France; ${ }^{4}$ Department of \\ Nephrology, University Hospital of Regensburg, Germany; \\ ${ }^{5}$ Nephrology Center, Medical Clinic and Polyclinic IV, \\ University of Munich, Munich, Germany; ${ }^{6}$ Institute of \\ Pathology, RWTH, Aachen, Germany
}

The recent advent of new sequencing technologies has revolutionized our view of the organization, activity and regulation of the human genome. Most importantly, it has now become evident that the majority of the genome is transcribed producing vast numbers of formerly unknown regulatory RNA species with significant involvement in human diseases. To this end, we have recently identified a large number of differentially expressed lncRNAs in the murine model of obstructive nephropathy UUO (Arvaniti et al, Scientific Reports 6:26235, 2016). Here, we provide evidence suggesting the involvement of one of these lncRNAs, human long intergenic noncoding RNA 01187 (LINC01187) and its mouse homolog (Gm12121-long) in human and rodent nephropathies. In particular, we show that the murine homolog, GM12121 long, exhibits a reduction in expression following ligation in the UUO mouse model. Moreover, chromatin immunoprecipitation assays revealed a concomitant reduction in the recruitment of RNA polymerase II and histone 3 tri-methylation in Lysine 4 (H3K4me3) on the promoter of GM12121-long in this model, indicating direct repression at the chromatin/transcription level. Similar reduction in expression was also observed in two other murine models, the anti-GBM and the ischemiareperfusion. These results, along with the high conservation of the promoter sequence among species and the fact that the expression of both murine and human homo- logs are reported to be highly renal specific (data from ENCODE and GTEx databases), prompted us to focus on the role of human homolog in renal pathologies. Thus, detailed examination of LINC01187 expression levels in human samples from different sources and pathological situations shows a significant reduction of its expression in diseased versus healthy kidneys. In addition, interrogation of its gene expression in microdissected renal biopsies of the European Renal cDNA Bank (ERCB) confirmed major reduction in expression in the cases of rapid progressive glomerulonephritis and diabetic nephropathy, both in the glomerular and the tubular compartment. Furthermore, by in situ hybridization studies using healthy and adjacent cancerous renal tissue from human biopsies, we observed strong expression of LINC01187 in elongated peri-glomerular cells, peri-tubular cells and certain glomerular cells in healthy tissue as well as total lack of expression in vicinal pathological tissue. Collectively, these data suggest a negative association of LINC01187 expression with renal pathologies that may imply a protective function.

\section{4 \\ Notch3 is Upregulated in Lupus Nephritis and Deficiency Accelerates SLE Progression}

\author{
Daniel Breitkopf ${ }^{1}$, Thomas Rauen ${ }^{1}$, Daniela Hermert ${ }^{1}$, \\ Elisabeth Gröne ${ }^{2}$, Ina Martin' ${ }^{1}$ Jürgen Floege', \\ Tammo Ostendorf ${ }^{1}$, Ute Raffetseder ${ }^{1}$ \\ ${ }^{1}$ Department of Clinical Immunology and Nephrology, \\ University Hospital RWTH, Aachen, Germany; ${ }^{2}$ Department of \\ Cellular and Molecular Pathology, German Cancer Research \\ Center, Heidelberg, Germany
}

Systemic lupus erythematosus (SLE) is a chronic inflammatory disease that affects the kidneys in about $50 \%$ of patients. Lupus nephritis is a major risk factor for overall morbidity and mortality in SLE. Notch signaling constitutes an evolutionarily conserved pathway that determines major decisions in cell proliferation, survival, and differentiation. To address the role of Notch-3 in SLE, we first measured the renal expression of Notch-3 in kidneys of SLE patients and in lupus-prone MRL.lpr mice. To test the role of Notch-3 in lupus, we crossbred Notch-3-/- and B6.Fas ${ }^{\text {pr/lpr mice }}$ to generate the B6.Fas ${ }^{\mathrm{lpr} / \mathrm{lpr}}$ Notch-3-/- genotypes and analyzed the spontaneous disease manifestations in 40 week-old mice. Furthermore, we administered antisense oligodeoxynucleotides (ODNs) targeting Notch-3 or scrambled controls in the more aggressive lupus-prone mice strain (MRL.Fas ${ }^{\mathrm{lpr} / \mathrm{lpr}}$ ) mice starting at week 12 for 3 weeks and analyzed these mice at the age of 16 weeks. In 20 week-old MRL.lpr mice, receptor Notch-3 and its target gene Hes2 were significantly up-regulated in kidneys in comparison to 8 and 12 week-old littermates. De novo renal Notch3 expression in lupus mice and SLE patients was especially pronounced in the glomeru-

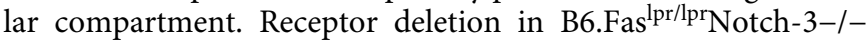
mice exhibited an aggravated lupus phenotype with earlier mortality, increased lymph nodes/spleen size, enhanced glomerular collagen IV deposition and increased immune cell infiltration in the kidneys. In line with this, application of antisense Notch-3 ODNs in MRL.lpr mice at the time of the transition from clinically inapparent to a manifested disease state (starting at week 12), resulted 
in accelerated disease progression as evidenced by enhanced lymphadenopathy, splenomegaly and an increase of inflammatory markers. Overall, these results demonstrate that Notch-3 exerts protective effects in the course of SLE, and its deficiency or inhibition accelerates the development of SLE manifestations.

\section{5}

Update About PEARL: Pathway Exploration and Analysis in Renal Lupus in the Accelerating Medicine Partnership (AMP) Phase 1 Lupus Network

\author{
Celine C. Berthier ${ }^{1}$, Arnon Arazi ${ }^{2}$, Deepak A. Rao ${ }^{3}$, \\ Edward P. Browne ${ }^{2}$, Thomas Eisenhaure ${ }^{2}$, Nir Hacohen', \\ David Lieb ${ }^{2}$, Betty Diamond ${ }^{4}$, Matthias Kretzler ${ }^{1}$, all the PEARL \\ team members \\ ${ }^{1}$ University of Michigan, Ann Arbor, MI, USA; ${ }^{2}$ Broad Institute, \\ Cambridge, MA, USA; ${ }^{3}$ Brigham and Women's Hospital, \\ Boston, MA, USA; ${ }^{4}$ Feinstein Institute for Medical Research, \\ Manhasset, NY, USA
}

Despite treatments, a substantial proportion of lupus nephritis (LN) patients progress to end stage renal disease and death. In 2014, the National Institute of Health (NIH), industry and nonprofit organizations joined their efforts with the AMP project, whose goal is to identify new diagnostic and therapeutic targets through a better understanding of the mechanisms by which individual cell types contribute to autoimmune tissue damage.

Renal biopsies from $24 \mathrm{LN}$ patients and 10 pre-transplant living donors (LD) were acquired across a distributed research network using a single, uniform pipeline developed by the AMP network. In brief, biopsies were cryopreserved and shipped to a centralized processing site for tissue dissociation. A total of 3,541 leukocytes and 1,621 epithelial cells were sorted from LN kidney samples. 438 leukocytes and 572 epithelial cells were sorted from LD biopsies. The transcriptome of those LN single tissue-infiltrating cells were assessed using single cell RNA-seq.

Extracted renal cells were clustered based on their gene expression data. A total of 20 immune cell populations active in the inflamed kidney were identified, including a majority of T-cell subsets (e.g. CD4 T-cells, CD8 T-cells). The distribution of clusters differed substantially between LN patients and controls. LD kidneys had no detectable B cells of any type. The frequent clusters in LD biopsies were those independently identified as resident tissue cells. Gene expression in cells from LN patients compared to those in LD highlighted unique LN dysregulated genes/pathways, such as the expected IFN. Two chemokine receptors (CXCR4 and CX3CR1) were expressed on the surface of almost all infiltrating immune cells and thus may be new therapeutic targets. CD45+ cells were isolated from LN urine samples. Compared to kidney, urine cells had major differences in frequencies of clusters including many more monocytes/macrophages. Renal and urine signatures significantly correlated, suggesting that urine cells could be used as potential markers for longitudinal monitoring and assessment of kidney inflammation and may be used to surrogate kidney biopsy.

The PEARL-Phase 0 project established protocols for renal tissue preservation, transport, processing and showed the feasibility of single cell isolation and transcriptomic analysis from LN kidney and urine. Results from the AMP-PEARL Phase 1 study identified LN active cells and pathways that can be used to guide the development of novel therapies. Analyses at a bigger scale $(n=200 \mathrm{LN})$ in Phase 2 will allow to correlate patterns and signatures of infiltrating cells with those of intrinsic renal cells, particularly the epithelial cells that make up $90 \%$ of renal cells and that are prone to hypoxic damage and cellular stress. It will accelerate the discovery of new therapeutic targets and identification of biomarkers to guide therapeutic decisions in $\mathrm{LN}$ and integrate the treatment effect.

\section{6 \\ Long-Term Kidney Function 3.5 Years after STEC-HUS Induced Kidney Injury}

\section{A. Spellmann, J. Steinhoff, M. Nitschke, l. Derad}

Nephrophrologie und Transplantation, Med. Klinik 1, Universität zu Lübeck, Lübeck, Germany

In 2011, during the northern German STEC-HUS outbreak 56 patients with severe hemolytic uremic syndrome had been treated in our center, first during their acute disease and thereafter for a follow-up in our outpatient clinic. Three years later, the patients were invited to answer a questionnaire and take part in a medical examination, with special regard to renal, hematological, neurological functions, development of hypertension and psychosocial well-being. The data were described and compared with the results after onset of the acute disease using Chi square, $\mathrm{u}$-test or t-tests as appropriate for comparison of the outcome parameters.

After a mean of 180 weeks data of 42 patients were recruited (45 patients after 56 weeks). Serum creatinine was elevated in $14.4 \%(4.4 \%$ after 56 weeks) with a mean creatinine of $78.0 \pm$ $14.1 \mu \mathrm{mol} / \mathrm{l}$, cystatin C was increased in $11.4 \%$ (26.7\% after 56 weeks). Albuminuria occurred in $11.4 \%$ (26.7\% after 56 weeks) and GFR according to the CKD-EPI formula was moderately reduced in $73.8 \%$ (46.7) with a mean of $82.2 \pm 17.5 \mathrm{ml} / \mathrm{min} / 1.73 \mathrm{~m}^{2}$. Kidney function of the cohort rather improved over time and nobody needed renal replacement therapy. The most prominent risk factor for a reduced kidney function was an older age at the onset of acute HUS. $25 \%$ of the patients had developed chronic hypertension. A higher creatinine during acute HUS, neurologic symptoms and a higher body mass index were identified as risk markers. During the follow-up two patients developed neurologic complications (TIA).

Conclusions: 3-4 years after the STEC-HUS outbreak patients had recovered from their severe disease and kidney function rather improved or kept stable. As a limitation only patients from one northern German centered were examined and data from the whole cohort should be analyzed. 


\section{7}

\section{Arg Tyrosine Kinase, TGF $\beta 1$ and Lysyl Oxidase Interactions in Clear Cell Renal Cell Carcinoma Progression}

\author{
S. De Marco ${ }^{1}$, C. Bianchi ${ }^{1}$, B. Torsello ${ }^{1}$, S. Bombelli ${ }^{1}$, N. Zucchini ${ }^{2}$, \\ P. Viganò ${ }^{3}$, R. Perego ${ }^{1}$
}

${ }^{1}$ School of Medicine and Surgery, University of Milano-Bicocca, Monza, Italy; ${ }^{2}$ Anatomo-Pathology Unit, ASST Monza, San Gerardo Hospital, Monza, Italy; ${ }^{3}$ Urology Unit, ASST North Milan, Bassini Hospital, Cinisello B, Italy

About $30 \%$ of clear cell Renal Cell Carcinoma (ccRCC) patients develops bone metastases, and an involvement of TGF $\beta 1$ in promoting these metastases has been described. Even the extracellular matrix enzyme Lysyl oxidase (Lox) is involved in formation of premetastatic bone lesions of breast cancer, through the activation of osteoclasts and the inhibition of osteoblasts. Our recent data evidenced that Lox is overexpressed in ccRCC and TGF $\beta 1$ production is modulated by Arg tyrosine kinase in human renal tubular cells under high glucose conditions. Arg is also involved, through cytoskeleton modulation, in the development of invasion and metastasis in breast and prostate cancer. All these data suggest that Arg, TGF $\beta 1$ and Lox might be functionally related to promote tumor invasion and metastasis. The aim of our study was to evaluate the molecular and functional interactions among Arg, TGF $\beta 1$ and Lox in ccRCC and to study their impact on osteoclast- and osteoblastdependent formation of pre-metastatic bone lesions. Our study took advantage of an in vitro model of primary cell cultures, obtained from specimens of ccRCC and matched normal renal cortex and characterized by FACS analysis, and ccRCC cell lines. TGF $\beta 1$ and Lox were significantly more abundant in media of low-grade ccRCC primary cultures than in those of high-grade ccRCC and normal cortex, as evaluated by ELISA. Even Arg protein, evaluated by western blot, was upregulated in low-grade with respect to highgrade ccRCC and normal cortex primary cultures. In 786-O ccRCC cell line the treatment with $5 \mathrm{ng} / \mathrm{ml}$ TGF $\beta 1$ for 24 hours induced an activation of Smad pathway, evaluated by western blot analysis of phospho-Smad 2 and Smad2/3 protein, and an increase of Lox secretion. Moreover, Arg silencing by siRNA in 786-O ccRCC cells increased TGF $\beta 1$ and Lox secretion and activated Smad pathway. We are now evaluating Lox secretion by $786-\mathrm{O}$ cells treated with Argsilenced ccRCC cell media in presence or absence of the TGF $\beta$ receptor inhibitor SB431542. The osteoclastic differentiation/activation of Raw264 macrophage cell line and the proliferation of MC3T3-E1 osteoblast cell line after treatment with conditioned media of Arg-silenced ccRCC cells will be also evaluated by TRAP staining and MTT assay, respectively. Our data highlighted that Arg expression is correlated with TGF $\beta 1$ and Lox production in low- and high-grade ccRCC primary cell cultures. Moreover, the effects of TGF $\beta 1$ treatment and Arg silencing in 786-O ccRCC cell line suggest an involvement of Arg in regulation of TGF $\beta 1$ secretion that, through Smad pathway activation, seems to increase Lox secretion. The eventual osteoclast activation and osteoblast inhibition induced by Arg-silenced ccRCC cell conditioned media, if confirmed by our ongoing analyses, might suggest that the complex interactions among Arg, TGF $\beta 1$ and Lox in ccRCC are important also for bone pre-metastatic niche formation.

\section{8 \\ Peritubular Capillary Loss Occurs in the First Month After Kidney Transplantation}

\author{
Anke A. Keijbeck ${ }^{1}$, Floor M.E.G. Steegh ${ }^{1}$, \\ Mariëlle A.C.J. Gelens ${ }^{2}$, Ernst L.W. van Heurn ${ }^{3}$, Erik A.L. Biessen", \\ Maarten H.L. Christiaans ${ }^{2}$, Carine J. Peutz-Kootstra ${ }^{1}$
}

Department of ${ }^{1}$ Pathology, ${ }^{2}$ Internal Medicine, Maastricht University Medical Centre, Maastricht, The Netherlands; ${ }^{3}$ Department of Surgery, Academic Medical Centre, Amsterdam, The Netherlands

Background: Peritubular capillary (PTC) density is associated with renal function in animal models and human renal biopsies. We have shown previously that a decrease of PTC density occurs in the first three months after transplantation in association with delayed graft function (DGF) and rejection [1]. We now further investigated PTC density decrease in these two groups by studying indication biopsies taken the first month after transplantation in an extended cohort.

Methods: Between August 2003 and December 2009, in 102 patients an indication biopsy was performed the first month after renal transplantation. These patients had also undergone protocolar biopsies at baseline and after 3 and 12 months. PTC density was studied as described earlier [1], and histological scoring was performed according to Banff criteria.

Results: In the DGF group $(n=66)$ there were more donation after circulatory death (DCD) donors than in the rejection group $(\mathrm{n}=36)(74.2 \%$ vs. $11.1 \%$, respectively). In the whole cohort, a significant decrease of PTC density was found in the indication biopsies, compared to baseline $(\mathrm{t}=6.42, \mathrm{p}<0.01)$, which was due to a decrease in PTC number $(\mathrm{t}=5.40, \mathrm{p}<0.01)$, while tubular numbers were stable $(t=0.40, p=0.69)$. When comparing the two subgroups, PTC density decrease in the first month after transplantation was more pronounced in patients with rejection than in patients with DGF. In the rejection group there was a stabilisation of the PTC density between 1 and three months $(t=0.65, p=0.52)$, concomitant with decreased inflammation $(\mathrm{t}=2.12, \mathrm{p}=0.04)$, which may be a treatment effect. In contrast, in the DGF group decrease of PTC density is enhanced in the 3-month biopsy $(\mathrm{t}=$ $3.64, \mathrm{p}<0.01)$ in association with increased total inflammation $(\mathrm{t}=$ $2.85, \mathrm{p}=0.006)$. In the biopsy after 12 months there was more interstitial fibrosis and tubular atrophy (IF/TA) in the DGF group vs. rejection group $\left(\mathrm{X}^{2}=9.04, \mathrm{p}=0.029\right)$.

Conclusions: Our results indicate that both ischemic and alloimmune injury are associated with decreased PTC density the first month after transplantation. In patients with DGF, decrease of PTC density aggravates over time in association with enhanced inflammation and more interstitial fibrosis after one year. Prevention of microvascular damage during and early after transplantation may be crucial to prevent chronic transplant dysfunction.

\section{Reference}

1 Steegh FM, Gelens MA, Nieman FH, van Hooff JP, Cleutjens JP, van Suylen RJ, Daemen MJ, van Heurn EL, Christiaans MH, Peutz-Kootstra CJ: Early loss of peritubular capillaries after kidney transplantation. J Am Soc Nephrol 2011;22(6):1024-1029. 


\section{9}

\section{K-Cadherin Release: A Precision System for a Repair Response to Injury Through Trans-Nephron Signalling}

Kameljit Kalsi ${ }^{1}$, Viji Draviam² ${ }^{2}$ Seema Jain ${ }^{1}$, Tri Nguyen ${ }^{3}$, Roel Goldshmeding ${ }^{3}$, Mark Dockrell ${ }^{1}$

${ }^{1} \mathrm{SWT}$ Institute for Renal Research, London, UK; ${ }^{2} \mathrm{School}$ Of Biological and Chemical Sciences Queen Mary University, London, UK; ${ }^{3}$ Department of Pathology University Medical School, Utrecht, The Netherlands

Introduction: Cadherin are highly conserved transmembrane proteins that play an instrumental role in morphogenesis by mediating cell:cell adhesion. The extracellular domain classically forms homophilic bonds with cadherins of adjacent cells while the intracellular domain binds indirectly to the actin cytoskeleton, thus creating links of the epithelial chain. In the renal tubule cadherins are considered "gate-keepers" of the epithelial phenotype with their loss or disruption associated with reduced function, epithelial mesenchymal transition and carcinogenesis. The human proximal tubule has a distinct cadherin expression profile; different from other regions of the tubule and different from the murine proximal tubule. Our previous work identified expression of $\mathrm{N}$ cadherin (cadherin 2) and K-cadherin (cadherin 6) in proximal tubule epithelial cells (PTEC) and E-cadherin (cadherin 1) in the distal tubule and collecting duct of samples from kidney donors. We also reported that elevated urinary full length K-cadherin from diabetic patients was an indicator of progressive decline of renal function. Our subsequent work seeks to investigate the function of $\mathrm{K}$-cadherin in health and disease.

Methods: Protocol biopsies from transplant patients and from prospective organ donors were probed for K-Cadherin by immunohistochemistry. HKC clone 8 cells, a transformed human PTEC line which do not have detectable K-cadherin protein, were grown on collagen IV coated coverslips and transfected with human Kcadherin pcDNA3.1 expression vector $(1 \mu \mathrm{g})$. After $48 \mathrm{~h}$ cells were fixed and immunostained for K-cadherin. Cells were visualized by using a 3D deconvolution microscope. Epithelial cells isolated from adult mice kidneys which do not have detectable K-cadherin protein were separated into predominantly distal and proximal fractions and cultured to confluence. Conditioned human PTEC media taken from confluent primary human PTEC cells and subjected to exosomal isolation was added to mice cells and incubated for $24 \mathrm{~h}$; cells treated with conditioned media were compared to cells treated with control media. Media was analysed for Bone Morphogenetic Protein 7 (BMP-7).

Results: Analysis of biopsy tissue confirmed the proximal tubule expression of K-Cadherin in 'donor' kidneys, with basolateral expression. In the transplant biopsies some PTEC had disrupted K-Cadherin and vesicular K-Cadherin was noted in some epithelial cells of distal tubules. In the transfected HKC8 cells Kcadherin was localised both in vesicular Golgi-type structures as well as at the cell periphery, predominantly in the basal layer. In media of primary human PTEC cultures we identified full length $\mathrm{K}$-Cadherin which remained after exosomal isolation. We subsequently investigated the effects of the K-cadherin containing conditioned media on preparations of mice primary cultures of "pre- dominantly distal" (a) or "predominantly proximal" (b) tubular epithelial cells and murine collecting duct cells, IMCD. Significant de novo $\mathrm{K}-\mathrm{Cadherin}$ protein was detected in distal mice cells treated with PTEC media compared to control media $(p<0.05, \mathrm{n}=6)$, $\mathrm{K}$-cadherin in mice proximal cells (megalin positive) treated with PTEC media was not as pronounced. No K-cadherin uptake was detectable in mice collecting duct cells. BMP-7 was released from both mice distal and proximal tubular cell cultures treated with PTEC media but not control media.

Conclusions: K-cadherin localisation in transfected HKC8 cells and healthy tissue is consistent with both a basement membrane sensor of the changes of matrix and a constituent of vesicles prepared for secretion. The up-take of K-cadherin by the treated murine distal cells is also consistent with our findings in protocol biopsy tissue from transplant recipients. This and the associated BMP-7 secretion strongly support our hypothesis of a previously unrecognised trans-nephron communication system.

\section{0 \\ A Novel Endothelial Cell Based Complement Dependent Cytotoxicity Test in Kidney Transplantation \\ R.G.M. Lammerts ${ }^{1}$, J. van den Born ${ }^{1}$, W.A. Dam ${ }^{1}$, M.A.J. Seelen ${ }^{1}$, B.G. Hepkema ${ }^{2}$, B.J. Kroesen ${ }^{2}$, M.R. Daha ${ }^{1}$, R.A.Pol', S.P. Berger ${ }^{1}$, on behalf of the COMBAT Consortium \\ 'Department of Nephrology, University Medical Centre Groningen, University of Groningen, Groningen, Netherlands; ${ }^{2}$ Department of Immunology, University Medical Centre Groningen, University of Groningen, Groningen, Netherlands; ${ }^{3}$ Department of Surgery, University Medical Centre Groningen, University of Groningen, Groningen, Netherlands}

Introduction: Relevant alloantibodies in kidney transplantation comprise anti-HLA antibodies, blood group antibodies (ABO incompatible) and anti-endothelial cell antibodies (AECA) and can initiate antibody-mediated rejection (ABMR). ABMR is characterized by complement activation products, such as $\mathrm{C} 4 \mathrm{~d}$, on the endothelium of the microvasculature in the kidneys at the time of rejection. However, endothelium directed cytotoxicity of the various antibodies and the role of complement (regulation) are not fully elucidated.

Methods: In this study, we set up a flow cytometry method and an endothelial complement dependent cytotoxicity test (EC-CDC) to evaluate the involvement of various transplant related antibodies in the process of complement mediated endothelial damage. We used primary endothelial cells (EC) cultured from donor kidney perfusate after machine perfusion, circulating human EC progenitors or conditionally immortalized human glomerular EC. Antibody binding and complement activation was evaluated by FACS analysis and compared to classical donor lymphocyte CDC (L-CDC) and EC-CDC.

Results: First, $\mathrm{ABO}$ incompatible serum caused complement mediated cell cytotoxicity in the EC-CDC. Second, sera containing high titer HLA-DSA that tested negative in the L-CDC, caused complement dependent cytotoxicity in the EC-CDC. This correlated with increased IgG binding and activation of $\mathrm{C} 3$ by flow cy- 
tometry. Serum, suspected to contain AECA, caused abundant cell death in the EC-CDC whereas no cytotoxicity was seen in the LCDC.

Conclusions: We successfully developed an EC-CDC and flow cytometry method to assess complement dependent endothelial cell damage and thereby show DSA mediated cytotoxicity and cytotoxicity of allo-antibodies that were undetectable by the classical L-CDC method, confirming the potential value of endothelial cell based cross-matching in transplantation.

\section{1 \\ Detection of Renal Allograft Rejection by NMR-Based Urine Metabolomics \\ Miriam Banas ${ }^{1}$, Sindy Neumann ${ }^{2}$, Philipp Pagel ${ }^{2}$, Dominik Chittka ${ }^{1}$, Volker Pfahlert ${ }^{2}$, Bernhard Banas ${ }^{1}$ \\ ${ }^{1}$ Department of Nephrology University Hospital Regensburg, Regensburg, Germany; ${ }^{2}$ Numares, Regensburg, Germany}

Introduction and Background: Post-transplant surveillance for acute rejection is typically based on regular monitoring of serum creatinine levels and consecutive biopsies upon functional renal impairment. Attempts have been made to develop non-invasive tests based on urinary markers to detect acute rejection. Probably due to high costs of analysis and lack of platform standardization, none of the tests has been established in the clinical routine so far. Recently, we explored an approach to detect rejection by a urinary metabolite network using inexpensive and standardized nuclear magnetic resonance spectroscopy (NMR). Our aim was to validate the test in a first prospective study.

Methods: Within the prospective Umbrella study performed at the University Hospital Regensburg 2,479 urine specimens from 109 consecutive kidney-graft recipients from day 1 through month 12 after transplantation were collected. The specimens were analyzed using NMR and the results were compared to the allograftrejection status according to the biopsy results.

Results: The metabolic constellation was able to detect acute cellular allograft rejection during outpatient phase ( $\geq$ day 15 after transplantation, area under the curve (AUC) 0.75 , [95\% confidence interval (CI) 0.68 to 0.83 ], $\mathrm{P}<0.001$ ). A combination of the test with serum creatinine based estimated glomerular filtration rate (eGFR) significantly improved the overall performance (AUC 0.84 ). In order to investigate possible drug interference effects on the metabolite network, we analyzed the score distribution in all available control urine samples from patients that never showed rejection episodes. In control urine samples only glucose infusions or antihistamine medication caused potential drug interferences. None of the other medications induced relevant increases in the score.

Conclusions: In conclusion, the metabolite constellation in combination with eGFR appears to be a reliable non-invasive test to support post-transplant outpatient biopsy decision making. To confirm the results from the previous Umbrella study we have now started a European multicenter study.

\section{2 \\ The Role of the Cannabinoid Receptors in Chronic Allograft Dysfunction}

\author{
Myriam Dao ${ }^{1}$, Lola Lecru ${ }^{3}$, Christos Chatziantoniou', \\ Hélène François ${ }^{1,2}$ \\ ${ }^{1}$ Inserm UMR_S 1155, Hôpital Tenon, Paris, France; ${ }^{2}$ AP-HP; \\ Service de médecine interne et immunologie clinique, Hôpital \\ Bicêtre, Université Paris Sud, Le Kremlin Bicêtre, France; \\ ${ }^{3}$ Galapagos SASU, Romainville, France
}

Rationale: The progressive and inevitable impairment of renal graft function called chronic allograft dysfunction (CAD) corresponds to replacement of functional renal tissue by extracellular matrix proteins leading to both, tubular atrophy and interstitial fibrosis. CAD is a complex and multifactorial process and a better understanding of renal fibrogenesis during CAD is an important goal. Our group has previously demonstrated a pro-fibrotic role of cannabinoid receptor 1 (CB1) in experimental renal fibrosis in the Unilateral Ureteral Obstruction (UUO) model and suggested that the CB1 pathway may be a new target in renal fibrosis. The aim of our study was to investigate the role of the cannabinoid system in renal fibrosis during CAD.

Materials and Methods: In the first part of our work, we retrospectively quantified CB1 expression by immunohistochemistry and morphometry software, and we correlated it with renal fibrosis in 26 kidney transplanted patients who underwent serial routine kidney biopsies at day 0 , month 3 and month 12. Additional data included Banff scoring, quantification of interstitial fibrosis (using Sirius Red staining followed by a morphometry software) and the usual clinical parameters. In the second part, we studied the effect of calcineurin inhibitors in CB1 expression using cell cultures of immortalized human proximal tubular epithelial cells (HK2) and of murine primary proximal epithelial tubular cells.

Results: CB1 expression is very low in normal kidney grafts, and is highly expressed in tubular epithelial cells, medium-sized arteries, interstitial inflammatory infiltrate and glomeruli during CAD. CB1 expression is significantly increased early on after transplantation, from D0 to M3 $(22.5 \pm 15.4 \%$ vs $33.4 \pm 13.8 \%$, p < $0.01)$ and it remains stable thereafter $(33.3 \pm 19 \%$ at $\mathrm{M} 12, \mathrm{p}=0.7$ vs M12). A positive correlation exists between $\mathrm{CB} 1$ expression and renal fibrosis at $M 3(p=0.035)$. In contrast, $C B 1$ expression is not correlated to glomerular filtration rate (GFR) at M3 and M12. Tacrolimus significantly increased CB1 expression in HK2 cells in vitro after $48 \mathrm{~h}$ of treatment ( $10 \mathrm{ng} / \mathrm{mL}$ daily) as evaluated by western blot analysis $(3.5 \pm 3.4$ vs $1.0 \pm 0, \mathrm{n}=4, \mathrm{p}=0.03)$. Similarly, tacrolimus increased expression of $\mathrm{CB} 1$ receptor in murine primary cultured tubular cells which was followed by activation of col1, col3 and col4 expressions. Interestingly, concomitant administration of tacrolimus with rimonabant, a CB1 antagonist, blunted the activation of collagens $(\mathrm{p}<0.05)$.

Conclusions: CB1 is increased early on in kidney grafts, is mostly localized in tubules and correlates with interstitial fibrosis. Tacrolimus is one of the factors inducing CB1 tubular expression and fibrosis in CAD. Treatment with a $\mathrm{CB} 1$ antagonist inhibits the fibrogenetic effect of tacrolimus. These data suggest an involvement of the cannabinoid system during CAD and highlight the therapeutic potential of $\mathrm{CB} 1$ antagonists. 
43

\section{Hemodialysis-Related Changes in Phenotypical Features of Monocytes}

Vassilios Liakopoulos ${ }^{1,3}$, Andreas Jeron ${ }^{2}$, Aneri Shah ${ }^{3}$, Dunja Bruder ${ }^{2,4}$, Peter R. Mertens ${ }^{3}$, Xenia Gorny ${ }^{3}$

${ }^{1}$ Division of Nephrology and Hypertension, 1st Department of Internal Medicine, AHEPA Hospital, School of Medicine, Aristotle University of Thessaloniki, Thessaloniki, Greece; ${ }^{2}$ Infection Immunology, Institute of Medical Microbiology, Infection Control and Prevention, Ottovon-Guericke University, Magdeburg, Germany; ${ }^{3}$ Clinic of Nephrology and Hypertension, Diabetes and Endocrinology, Otto-vonGuericke-University Magdeburg, Leipziger Str. 44, 39120 Magdeburg, Germany; ${ }^{4}$ Immune Regulation, Helmholtz Centre for Infection Research, Braunschweig, Germany

Background: Patients with end-stage-renal-disease undergoing hemodialysis (HD) exhibit chronic inflammation, which is mainly attributed to the accumulation of toxins and procedureassociated leukocyte activation. In this study we investigated the surface marker profile of monocytes to estimate and compare the chronic effect of uremia and the acute effect of the dialysis procedure on phenotypical features of monocytes.
Methods: Blood from $16 \mathrm{CON}$ and $15 \mathrm{HD}$ patients before (pre) and after (post) a dialysis session with polysulfone filters was stained with fluorochrome labeled antibodies followed by flow cytometric analysis. Expression of integrins (CD11b and CD18), chemokine receptors (CCR2 and CX3CR1), scavenger receptors (CD36 and CD163) and Toll-like receptor 2 (TLR2) on monocytes, indicated by the median fluorescence intensities (MFI), was quantified.

Results: Before and after dialysis HD patients bore less CD14+CD16- classical Mo1 and more CD14+CD16+ intermediate Mo2, and CD14dimCD16+ non-classical Mo3 monocytes compared to CON. Mo1 monocytes from HD patients showed elevated expression of CD11b, CD18 and CD36 and Mo1 and Mo2 monocytes showed reduced expression of CD163 compared to CON. All of these markers were unaffected by the dialysis procedure. In contrast, CX3CR1 expression on Mo2 and Mo3 monocytes was lower in HD patients before and further diminished after dialysis, whereas TLR2 expression was acutely enhanced comparing pre- and post-intervention conditions.

Conclusions: A systematic analysis of the expression of particular surface markers such as CX3CR1 and Toll-like receptors on monocyte subsets may help to distinguish between effects caused by uremia and/or dialysis procedures and to evaluate the biocompatibility of dialysis membranes. 


\section{Author Index}

\section{nephron \\ Experimental \\ Nephrology \\ and Genetics}

Number refers to abstract number
Abed, A. 27

Allinovi, M. 11

Amann, K. 29

Anders, H.-J. 5, 19

Anders, H.J. 6

Andrews, D. 31

Angelotti, M.L. 11

Antonelli, G. 11

Arazi, A. 35

Artelt, N. 28

Arvaniti, E. 33

Authier, F. 27

Averdunk, L. 3

Bachelier, R. 27

Ballhause, T. 15

Banas, B. 32, 41

Banas, M. 33, 41

Banas, M.C. 32

Bang, M.L. 28

Bardin, N. 27

Bäuerle, T. 19

Beck, S. 32

Becker, A. 15

Berger, S.P. 40

Bernhagen, J. 3

Bernhardt, A. 13, 15

Berthier, C.C. 35

Bianchi, C. 8, 16, 37

Biessen, E.A.L. 38

Blot-Chabaud, M. 27

Blumenthal, A. 30

Böhland, C. 19

Bolognesi, M. 8

Bombelli, S. 8, 16, 37

Boor, P. 3, 33

Botton, O. 18

Brandt, S. 13, 15

Braun, G. 22

Breitkopf, D. 14, 34

Brennan, E. 31

Browne, E.P. 35

Bruder, D. 43

Bucala, R. 3

Burtey, S. 27

Calmont, A. 10

Capusa, C. 20

Caron, N. 18

Cassina, V. 16
Cattoretti, G,, 8

Chadjichristos, C. 1, 2, 10

Chadjichristos, C.E. 27, 28

Charonis, A. 32, 33

Chatziantoniou, C. 1, 2, 10, $28,33,42$

Chen, P. 24

Chisci, E. 16

Chittka, D. 32, 41

Christiaans, M.H.L. 38

Coburn, M. 3

Cohen, C.D. 22, 29, 33

Conlon, P. 31

Conte, C. 11

Corchia, A. 26

Daha, M.R. 40

Dam, W.A. 40

Dao, M. 42

De Chiara, L. 11

De Marco, S. 8, 16, 37

Declèves, A.-E. 18

Derad, I. 36

Diamond, B. 35

Dignat-George, F. 27

Djudjaj, S. 3, 33

Dockrell, M. 39

Doyle, R. 31

Draviam, V. 39

Dussaule, J.-C. 1, 2, 10

Eckes, T. 21

Eisenhaure, T. 35

El Bounkari, O. 3

Endlich, K. 28, 29, 30

Endlich, N. 28, 29, 30

Federico, G. 7

Fehr, A. 15

Floege, J. 9, 14, 22, 34

Foucault-Bertaud, A. 27

François, H. 42

Fu, Y. 15

Gakiopoulou, H. 33

Gaussin, J.-F. 18

Gelens, M.A.C.J. 38

Godson, C. 31

Goetzenich, A. 3

Goldshmeding, R. 39

Gorny, X. 43

Grill, J.F. 19
Gröne, E. 7, 34

Gröne, H.-J. 7

Gröne, H.J. 22

Guo, J. 24

Hacohen, N. 35

Hadchouel, J. 4

Hamadache, S. 4

He, B. 24

Heidel, F. 15

Hepkema, B.G. 40

Hermert, D. 14, 34

Iosif, L. 20

Iwakura, T. 5, 6

Jadot, I. 18

Jain, S. 39

Jenart, V. 18

Jerchel, S. 13

Jeron, A. 43

Johannes, L. 7

Jordan, J. 19

Jourde-Chiche, N. 27

Juszczak, F. 18

Kaling, S. 29

Kalsi, K. 39

Kaltezioti, V. 33

Kavvadas, P. 1, 27, 28, 33

Keijbeck, A.A. 38

Kim, B.S. 3

Kliewe, F. 29

Knops, N. 17

Koch, A. 21

Kormann, R. 1, 2

Kraemer, S. 3

Kretz, M. 32

Kretzler, M. 35

Kriz, W. 23

Kroesen, B.J. 40

Kundert, F. 6

Kuypers, D.J. 17

Lammerts, R.G.M. 40

Lasagni, L. 11

Lazzeri, E. 11

Lech, M. 19

Lecru, L. 42

Legrand, A. 18

Leng, L. 3
Leroyer, A.S. 27

Levtchenko, E. 17

Liakopoulos, V. 43

Lieb, D. 35

Lindenmeyer, M.T. 22, 29, 33

Lindquist, J.A. 13

Linkermann, A. 3

Loetzsch, H. 29

Louis, K. 26

Luque, Y. 26

Ma, Q. 19

Ma, X. 25

Manolakou, D. 33

Marlier, A. 3

Maroteaux, L. 22

Marschner, J.A. 6

Martin, I. 34

Martin, I.V. 9, 14

Marx, G. 3

Mattiotti, M. 9

Mazzinghi, B. 11

McEvoy, C. 31

Mehedinti, A.-M. 20

Melica, M.E. 11

Meregalli, C. 8

Mertens, P.R. 13, 15, 43

Mesnard, L. 26

Mircescu, G. 20

Moeckel, G. 3

Moeller, M.J. 22

Morace, I. 7

Mueller Deile, J. 31

Müller, A. 15

Neumann, S. 41

Nguyen, T. 39

Niasse, A. 4, 26

Nitschke, M. 36

Ochi, A. 3

Ostendorf, T. 9, 14, 22, 34

Otey, C. 28

Pagani, M. 8

Pagel, P. 41

Patrakka, J. 24, 25

Peired, A. 11

Perego, R. 8, 16, 37

Peutz-Kootstra, C.J. 38

Pfahlert, V. 41 
Pfeilschifter, J. 21

Philipsen, L. 15

Placier, S. 1, 2, 4, 10, 26

Platen, L. 6

Pol, R.A. 40

Politis, P. 33

Prakoura, N. 1, 2

Raffetseder, U. 9, 14, 34

Ramazani, Y. 17

Ranzani, V. 8

Rao, D.A. 35

Rauen, T. 34

Reichelt-Wurm, S. 32

Reichert-Wurm, S. 33

Reiss, K. 14

Rex, S. 3

Ritter, A.M. 28

Rodrigues, P. 24

Romagnani, P. 6, 11
Rondeau, E. 26

Rossetti, G. 8

Saalmüller, K. 22

Sadlier, D. 31

Sandberg, R. 24

Scambler, P. 10

Scharf, C. 29

Schießl, I. 7

Schiffer, M. 31

Schindler, M. 30

Schlüter, R. 28

Schroder, P. 31

Schroeder, S. 29

Schwarz, A. 25

Seelen, M.A.J. 40

Sevilla, S.Z. 24, 25

Shah, A. 43

Smeets, B. 22

Smolle, M. 19
Soppert, J. 3

Sounidaki, M. 13

Spellmann, A. 36

Staggs, L. 31

Stancu, S. 20

Steegh, F.M.E.G. 38

Steiger, S. 19

Steinhoff, J. 36

Stevanovic, A. 3

Stoian, I. 20

Stoppe, C. 3

Surin, B. 10

Tassin, A. 18

The GENIE Consortium 31

Torsello, B. 8, 16, 37

Valssembrouck, M. 18

van den Born, J. 40

van den Brandt, J. 28 van den Heuvel, B.P. 17

van Heurn, E.L.W. 38

van Roeyen, C.R.C. 22

Vandermeersch, S. 2, 26

Verpont, M.-C. 2

Viganò, P. 8, 37

Vu, J. 22

Weiskirchen, R. 14

Wirtz, T. 32

Wolters, K. 13

Xu-Dubois, Y.C. 26

Ye, G. 10

Zarbock, A. 3

Zhao, Z. 6

Zucchini, N. 37

Zwakhals, T. 18 